\title{
Simultaneous MEG and intracranial EEG recordings during attentive reading
}

\author{
Sarang S. Dalal ${ }^{\mathrm{a}}$, Sylvain Baillet ${ }^{\mathrm{b}}$, Claude Adam ${ }^{\mathrm{b}}$, \\ Antoine Ducorps ${ }^{\mathrm{b}}$, Denis Schwartz ${ }^{\mathrm{b}}$, Karim Jerbi ${ }^{\mathrm{a}, \mathrm{c}}$, \\ Olivier Bertrand $^{\mathrm{a}}$, Line Garnero ${ }^{\mathrm{b}}$, Jacques Martinerie ${ }^{\mathrm{b}}$, \\ Jean-Philippe Lachaux ${ }^{\mathrm{a}, \mathrm{b}}$ \\ ${ }^{a}$ Dynamique Cérébrale et Cognition, INSERM Unité 821, Lyon, France; Institut \\ Fédératif des Neurosciences, Lyon, France; Université Lyon 1, France \\ ${ }^{\mathrm{b}}$ Laboratoire de Neurosciences Cognitives et Imagerie Cérébrale, CNRS \\ UPR640-LENA, Hôpital de la Salpêtrière, Paris, France; UPMC Univ. Paris 06, \\ Paris, France \\ ${ }^{\mathrm{c}}$ Laboratoire de Physiologie de la Perception et de l'Action, CNRS UMR 7152, \\ Collège de France, Paris, France
}

\begin{abstract}
The relationship between neural oscillations recorded at various spatial scales remains poorly understood partly due to an overall dearth of studies utilizing simultaneous measurements. In an effort to study quantitative markers of attention during reading, we performed simultaneous magnetoencephalography (MEG) and intracranial EEG (iEEG) recordings in four epileptic patients. Patients were asked to attend to a specific color when presented with an intermixed series of red words and green words, with words of a given color forming a cohesive story. We analyzed alpha, beta, and gamma band oscillatory responses to the word presentation and compared the strength and spatial organization of those responses in both electrophysiological recordings. Time-frequency analysis of iEEG revealed a network of clear attention-modulated high gamma band $(50-150 \mathrm{~Hz})$ power increases and alpha/beta (9-25 Hz) suppressions in response to the words. In addition to analyses at the sensor level, MEG time-frequency analysis was performed at the source level using a sliding window beamformer technique (Dalal et al., 2008). Strong alpha/beta suppressions were observed in MEG reconstructions, in tandem with iEEG effects. While the MEG counterpart of high gamma band enhancement was difficult to interpret at the sensor level in two patients, MEG time-frequency source reconstruction revealed additional activation patterns in accordance with iEEG results. Importantly, iEEG allowed us to confirm that several sources of gamma band modulation observed with MEG were indeed of cortical origin rather than EMG or ocular artifact.
\end{abstract}

Key words: magnetoencephalography, subdural electrodes, intracranial 
electroencephalography, electrocorticography, epilepsy surgery, gamma band, source localization, beamformer

\section{Introduction}

An increasing body of evidence gathered over the last fifteen years suggests that most cognitive functions, from sensory and motor processing to language or memory, involve the formation of local neural assemblies characterized by synchronous oscillations in the gamma band, i.e., at frequencies starting at $40 \mathrm{~Hz}$ (for reviews, see Singer, 1999; Tallon-Baudry and Bertrand, 1999). Interestingly, the formation of such assemblies seems to depend upon the degree of attention allocated by subjects or animals to the task at hand (Bauer et al., 2006; Bichot et al., 2005; Fries et al., 2001; Gruber et al., 1999; Steinmetz et al., 2000; Tallon-Baudry et al., 2005), which suggests that monitoring gamma band activity could provide a quantitative measure reflecting attention. Indeed, in a recent intracerebral EEG study, which manipulated explicitly the attention of subjects in a verbal working memory task, we found that gamma band responses were stronger for attended stimuli, later recalled, than for unattended ones (Mainy et al., 2007).

We have all experienced the effect of attention fluctuations on performance in the context of reading, sometimes having the sensation of "flying over" entire pages without truly absorbing their meaning. The previously mentioned studies led us to hypothesize that it may be possible to monitor the degree of attention during reading by measuring the spectral power in the alpha, beta, and gamma bands. Testing this hypothesis in healthy individuals assumes that attention-related power modulations in the lower alpha and beta bands as well as those in the gamma band can be detected with noninvasive recordings such as MEG or scalp level EEG. The present study tackles this issue by investigating the degree and precision by which gamma band power (and to a lesser extent, alpha and beta band) modulations of cortical activity can be recovered at the scalp level in individual subjects during attentive reading. The ability of surface-level electrophysiological measurements to detect gamma band responses (GBR) is still a matter of debate. Although it has been more than a decade since the first reports of gamma band power modulations at t he scalp level with EEG (e.g., Tallon-Baudry et al., 1996; Tallon-Baudry and Bertrand, 1999; Rodriguez et al., 1999; Müller et al., 2000), with MEG reports arriving more recently (e.g., Kaiser et al., 2002, 2004; Osipova et al., 2006; Vidal et al., 2006; Guggisberg et al., 2008; Dalal et al., 2008a), those

Email address: jp.lachaux@inserm.fr (Jean-Philippe Lachaux). 
responses have mostly been detected through population-level statistics. The relatively limited number of such reports has so far impeded the use of GBR as a research or clinical index even though increasing results from intracranial EEG suggest that high gamma band modulations could be used as functional markers (Lachaux et al., 2005, 2006; Sederberg et al., 2003; Szurhaj et al., 2005; Tallon-Baudry et al., 2005; Tanji et al., 2005) tightly related to task-related hemodynamic variations measured by fMRI (Kim et al., 2004; Niessing et al., 2005; Lachaux et al., 2007). In contrast, other important electrophysiological indices such as the N100 evoked potential and mismatch negativity (MMN) are routinely used for both research and clinical purposes. The absence of GBR from clinical use thus cannot be fully explained by the younger age of the field and certainly not to a lack of interest by the EEG/MEG community; more likely, it is due to acknowledged methodological difficulties associated with extracting gamma response components from noninvasive recordings (Trujillo et al., 2005; Hoogenboom et al., 2006).

Moreover, the failure to detect EEG/MEG GBR in a given subject can be a source of confusion. For example, the technical limitations of EEG/MEG could have prevented detection, due to a low signal to noise ratio in the gamma band or insufficient spatial resolution. Alternatively, it could have been the result of individual subject differences. For example, a subject may have employed a different strategy or been less attentive than the others, and therefore employed different cortical mechanisms that may not have involved GBR.

To evaluate our ability to noninvasively recover cortical GBR, and their modulation by attention during reading, we designed an experiment to simultaneously record MEG and intracerebral EEG (iEEG) responses in epileptic patients implanted with depth electrodes and subdural strips for clinical reasons. Such simultaneous recordings provided a unique way to compare the spectral content of the scalp traces of GBR, as well as lower-frequency modulations, to those recorded directly from the cortex via the implanted depth electrodes. It was considered more advantageous to record iEEG simultaneously with MEG as opposed to scalp EEG, due to the difficulty recording from EEG electrode caps in patients with intracerebral electrodes, as well as the likely considerable changes in electric field propagation caused by the implants and craniotomies (Kirchberger et al., 1998).

We simultaneously recorded both MEG and iEEG data in four patients while they performed a simple reading task with variable levels of attention depending on instructions on whether to attend to the narratives formed by the words. In addition to being a proof of concept of simultaneous MEG and iEEG data acquisition, this study presents and discusses the MEG correlates of strong intracerebral alpha, beta, and gamma power modulations generated by word stimuli in temporal, occipital, and parietal lobes. The experimental design is the same used in a comprehensive iEEG study from our group (Jung 
et al., 2008), and originally implemented by Nobre et al. (1998) for an fMRI experiment.

Previous human studies have described simultaneous acquisition of MEG and iEEG to evaluate high-amplitude epileptiform spikes (Mikuni et al., 1997; Sutherling et al., 2001; Shigeto et al., 2002; Oishi et al., 2002). However, to our knowledge, this experiment comprises the first successful simultaneous recordings to study cognitive function.

\section{Methods}

\subsection{Subjects}

Simultaneous MEG and iEEG recordings were obtained from four patients with intractable epilepsy (referred to as Pt1-Pt4) who were candidates for resective surgery. Table 1 summarizes their clinical, neuroimaging, and videoEEG characteristics. Intracranial electrodes were implanted in these patients for preresection seizure localization and functional mapping of critical language and motor areas. Electrode implants were guided strictly by clinical indications and research recordings were approved by the local institutional review board. Upon informed consent, the experiments were conducted while each patient was alert and on minimal medication. All patients were implanted with depth electrodes and, in the case of Pt1, additionally with subdural strips placed on the cortical surface. Depth electrodes were inserted stereotactically through the aid of a Leksell frame using an "orthogonal" approach; electrode strips were placed via burr holes, also precisely positioned using sterotactic coordinates (Dormont et al., 1997). The brain regions explored were bilateral temporal in $\mathrm{Pt} 1$, the whole right temporal lobe with its basal and posterior part in Pt2, the whole left temporal lobe with its junction to the occipital and parietal lobes in Pt3, and anterior temporal and frontal regions in the right hemisphere of Pt4.

\subsection{Electrode implantation}

Each patient was implanted with 7 to 9 linear electrode arrays. Each depth electrode array had a diameter of $1 \mathrm{~mm}$. Both depth electrode arrays and subdural strips contained 4 to 8 cylindrical contacts of $2.3 \mathrm{~mm}$ length, $10 \mathrm{~mm}$ apart center-to-center (Ad-Tech Medical, Racine, WI, USA). Therefore, various mesial and lateral cortical areas were evaluated, including sulcal cortex. A total of 174 sites were recorded from the four patients, with 68 in the left 
Table of Patient Characteristics

\begin{tabular}{|c|c|c|c|c|c|}
\hline Patient & $\begin{array}{l}\text { Gender/ } \\
\text { Age }\end{array}$ & $\begin{array}{l}\text { Surface Video-EEG } \\
\text { (seizure onset) }\end{array}$ & MRI & $\begin{array}{l}\text { Intracranial Location } \\
\text { of Seizure Onset }\end{array}$ & $\begin{array}{l}\text { Surgical Outcome } \\
\text { (Engel's class*) }\end{array}$ \\
\hline Pt1 & $\mathrm{F} / 33$ & $\mathrm{R}$ and $\mathrm{L}$ temporal & n.l. & $\begin{array}{l}\mathrm{R} \text { and } \mathrm{L} \text { neocortical } \\
\text { temporal (MTG) }\end{array}$ & not resected \\
\hline Pt2 & $\mathrm{F} / 28$ & $\begin{array}{l}\mathrm{R} \text { temporal (mostly } \\
\text { anterior) }\end{array}$ & n.l. & $\begin{array}{l}\mathrm{R} \\
\text { basal }\end{array}$ & IIIa \\
\hline Pt3 & $\mathrm{M} / 45$ & $\begin{array}{l}\mathrm{L} \text { posterior tempo- } \\
\text { ral and occipital }\end{array}$ & n.l. & $\begin{array}{l}\mathrm{L} \text { posterior temporal } \\
\text { basal }\end{array}$ & IV \\
\hline Pt4 & $\mathrm{M} / 39$ & $\begin{array}{l}\mathrm{R} \text { anterior frontal } \\
\text { and temporal }\end{array}$ & n.l. & $\mathrm{R}$ temporal pole & not resected \\
\hline
\end{tabular}

Table 1

Abbreviations: $\mathrm{L}=$ left; $\mathrm{R}=$ right; $\mathrm{MTG}=$ middle temporal gyrus; $\mathrm{n} . \mathrm{l} .=$ no lesion.

*from Engel (1987)

hemisphere and 106 in the right hemisphere. The electrode contacts were identified directly on the patients' MRI, then segmented and 3D-rendered using BrainVisa (http://brainvisa.info) and in-house MATLAB code.

\subsection{Paradigm}

An experimental paradigm similar to Nobre et al. (1998) was used, modified with slightly longer interstimulus intervals to adjust for the late latencies of the attentional modulations reported in that study. In each block of the experiment, the subjects were presented with 400 words shown individually at a rapid rate (words presented for $100 \mathrm{~ms}$ with an interstimulus interval of $800 \pm 100 \mathrm{~ms}$ ) on a black computer screen. 200 words were presented in green and the other 200 presented in red, with words of a given color forming a meaningful and simple short story in French. The subjects were instructed to read the story presented with a specified target color and be prepared to answer questions about the narrative after the block. The distribution of colors within the 400-word series was randomized, so that subjects could not predict whether or not the subsequent word was to be attended; the randomization was constrained to avoid series of more than 3 consecutive words with the same color. There were 6 blocks in total, and the target color was varied between blocks.

After each block, subjects were asked questions about the relevant story that could not have been answered from general knowledge. They were also asked to recount the presented story to the best of their ability. The analysis compared brain responses to attended words (i.e., words of the target color: TARGETS) versus brain responses to unattended words (i.e., words of the non-target color: DISTRACTORS). 
The experimental procedure took place in the shielded MEG testing room. Stimuli were presented to the participants on a 17" computer screen at a $90 \mathrm{~cm}$ viewing distance, and the average word subtended $3^{\circ}$ of visual angle.

Note that this particular paradigm had several important advantages for the present study: a) it was short (less than 30 minutes) and could fit in the tight MEG experimental schedule of the patients, b) it provided a large number of trials during that short time window, typically yielding over 200 trials for each condition, even after removing trials contaminated by artifacts such as epileptiform spikes or eye blinks, c) it was interesting for the patients and well-suited to maintain their attention, despite the large number of trials, d) finally, the task involved a widely distributed cortical network, which could likely be captured via a variety of implantation schemes, in contrast with, e.g., a purely motor task whose activated network could have only been studied in patients with motor cortex implants.

\subsection{Recording and data analysis}

\subsubsection{MEG Recordings}

The experiment was conducted at the Centre MEG of the Hôpital de la Salpêtrière, Paris, France. MEG recording was performed using a whole-head MEG system (VSM MedTech, Coquitlam, BC, Canada) with 151 radial gradiometers over the scalp and 29 reference gradiometers and magnetometers for ambient field correction. Signals were digitized at a sampling rate of $1250 \mathrm{~Hz}$ (0-200 Hz bandwidth) during epochs lasting $5 \mathrm{~s}$, beginning $1 \mathrm{~s}$ prior to stimulus onset. Eye movements were monitored with four ocular electrodes (Viasys Healthcare, Madison, WI, USA). Trials were rejected offline for eye blinks, eye movements, head movement, muscular, epileptiform, or electromagnetic artifacts. MEG data were coregistered with the structural MRI of each patient using BrainStorm (http://neuroimage.usc.edu/brainstorm).

\subsubsection{Intracerebral EEG recordings}

Intracerebral recordings were conducted using an EEG monitoring system (Micromed, Treviso, Italy), which allowed the simultaneous recording of 63 iEEG channels sampled at $1024 \mathrm{~Hz}(0.1-200 \mathrm{~Hz}$ bandwidth) during the experimental paradigm. A contact site in white matter was chosen as the reference electrode in each patient. This reference was ensured to have similar impedance to the other contact sites; in addition, it was verified to be free of contamination from eye movement artifacts and electromyographic activity from subtle muscle contractions. However, all electrodes were re-referenced to an adjacent electrode $(1 \mathrm{~cm}$ away) to form a bipolar montage. We used 
this montage to enhance the spatial resolution of the recordings and to ensure that the recorded activity was generated locally to the electrodes. In total, there were 44 such bipolar pairs for Pt1, 14 for Pt2, 27 for Pt3, and 23 for Pt4. Recording sites showing epileptiform activities were excluded from the analysis, and among the remaining sites, monopolar, and bipolar data were systematically inspected (first unfiltered, then highpass filtered at $15 \mathrm{~Hz}$ ), and any trial showing epileptic spikes in any of those traces was discarded. Note that this highpass filtering was done only for artifact rejection; all analyses shown in this paper were done with the raw, unfiltered signals.

Prior to signal analysis, all trials were sorted into two categories: targets (words to be attended) and distractors (words not to be attended) irrespective of their colors. In the final dataset after artifact rejection, we adjusted the size of the trial sets so that each trial category (target and distractor) would contain an equal number of red and green words.

\subsubsection{Evoked response analysis}

Evoked potentials and fields were computed by averaging each channel over all the trials of a given type (target or distractor), subtracting the mean of the baseline of period of ${ }^{-} 200$ to ${ }^{-} 50 \mathrm{~ms}$.

\subsubsection{Time-frequency analysis}

For each single trial, bipolar derivations computed between adjacent electrode contacts were analyzed in the time-frequency (TF) domain by convolution with complex Morlet's wavelets (Tallon-Baudry et al., 1996), thus providing a TF power map $P(t, f)=|w(t, f) * s(t)|^{2}$, where for each time $t$ and frequency $f, w(t, f)$ was a complex Morlet's wavelet $w(t, f)=A \mathrm{e}^{-t^{2} / 2 \sigma_{t}^{2}} \mathrm{e}^{2 \pi i f t}$, with $A=\left(\sigma_{t} \sqrt{\pi}\right)^{-1 / 2}$ and $\sigma_{t}=\left(\frac{2 \pi}{7} f\right)^{-1}$.

\subsection{Statistical analysis}

\subsubsection{Comparison with baseline level within a single condition}

For a given stimulus type (targets or distractors), significant spectral energy modulations (SSEM) caused by the stimuli (i.e., energy increase or decrease relative to pre-stimulus level) were detected using Wilcoxon matched paired nonparametric tests. We compared the total energy in a given time-frequency window with that of a tile of the same frequency extent across trials, but covering a $\left[{ }^{-} 200\right.$ to $\left.{ }^{-} 50 \mathrm{~ms}\right]$ pre-stimulus baseline period. The result of a Wilcoxon test is a $Z$ value which quantifies the energy variation relative to 
baseline for the chosen time-frequency tile. Wilcoxon tests were also used to draw statistical TF maps of $Z$ values representing the effects of stimulation in multiple adjacent $[40 \mathrm{~ms} \times 8 \mathrm{~Hz}] \mathrm{TF}$ tiles covering a large $\left[{ }^{-} 200\right.$ to $600 \mathrm{~ms}$ ] $\times$ [1-200 Hz] TF domain) (see Figures 2-6): such maps allow the direct visualization of SSEM in time and frequency for a given recording site. $Z$ values can be directly converted into $p$ values to quantify the risk associated with rejecting the null hypothesis of no change relative to baseline ( $p$ being only function of $Z$, independent of the number of trials). To correct for multiple testing (number of sites $\times$ number of non-overlapping TF tiles), we applied a Bonferroni correction yielding $p$ values for significance less than $3 \times 10^{-6}$ (Pt1), $9 \times 10^{-6}(\mathrm{Pt} 2), 5 \times 10^{-6}(\mathrm{Pt} 3)$, and $6 \times 10^{-6}(\mathrm{Pt} 4)$.

Since our goal was to test whether iEEG SSEM were visible in MEG, our strategy for analysis of MEG data consisted of 1) defining, from the previous analysis, TF windows with iEEG SSEM, and then 2) for those TF windows, test at each MEG sensor whether the MEG energy level was different from baseline level using Wilcoxon matched paired nonparametric tests. The outcome of the iEEG analysis (see Results) led us to investigate SSEM in MEG data in two TF windows $\left({ }^{+} 200\right.$ to $\left.{ }^{+} 500 \mathrm{~ms}\right] \times[9-25 \mathrm{~Hz}]$ for alpha/beta suppressions $(\mathrm{ABS})$ and $\left[{ }^{+} 100 \mathrm{~ms}\right.$ to $\left.{ }^{+} 400 \mathrm{~ms}\right] \times[50-100 \mathrm{~Hz}]$ for GBR). Wilcoxon tests were performed with and without Bonferroni correction [uncorrected threshold of $p=0.05$, and corrected threshold of $p=0.05 /(2 \times 151)$ to account for $2 \mathrm{TF}$ windows and 151 sensors].

\subsubsection{Comparison between responses to targets and distractors}

Differences between responses to targets and distractors were evaluated statistically in the TF plane using Mann-Whitney nonparametric tests using ELAN Pack, EEG/MEG analysis software developed by the INSERM U821 laboratory (http://www.lyon.inserm.fr/821/). For MEG data, there was one test for each sensor and each of the two TF windows defined above, again with two significance thresholds: uncorrected $p=0.05$, and corrected $p=0.05 /(2 \times 151)$. For iEEG data, there was one test for each recording site and each of the two TF windows defined above, with significance thresholds of $p=0.05$ uncorrected, and $p=0.05 / M$ corrected, where $M$ is the number of recording sites $(M=44$ for Pt1, 14 for Pt2, 27 for Pt3, and 23 for Pt4).

\subsubsection{Cross-sites synchrony analysis}

Cross-sites synchrony was evaluated using wavelet-based estimation measuring the degree of phase-locking between certain spectral components of the response (alpha, beta, and gamma responses) (Lachaux et al., 2002). In short, the analysis extracts the time course of the instantaneous phase-difference be- 
tween two signals in each trial around a frequency of interest (as computed using Morlet's wavelets), and estimates the circular variance of that difference on a sliding window the duration of which depends on the analyzed frequency (8 cycles of oscillations). This circular variance is a time-varying measure of phase-locking between 0 (no phase-locking) and 1 (complete synchrony) which is averaged across trials to provide an average measure of synchrony between signals.

\subsubsection{GBR dipole moment estimation}

The dipole moment of GBR sources detected with iEEG was coarsely estimated via a standard source-field model and the Hilbert transform. First, for each single trial, the bipolar iEEG data was bandpass filtered to $50-100 \mathrm{~Hz}$ (100-150 Hz for Pt4) and Hilbert transformed to extract the GBR envelope. These envelopes were then averaged across trials. The maximum value over the 300-700 ms interval for each bipolar channel was then noted.

The relationship between recorded voltage and dipole moment is given by the following equation (Malmivuo and Plonsey, 1995):

$$
\Phi \approx \frac{p \cos \theta}{4 \pi \sigma r^{2}},
$$

where $\Phi$ is the measured voltage potential at a given electrode, $p$ is the dipole moment, $r$ is the distance between the dipole and electrode, $\theta$ is the angle between the dipole axis and the electrode location, and $\sigma$ is the tissue conductivity. For the purpose of this estimate, we assume that a bipolar iEEG pair records a dipole located at the midpoint between the two locations and oriented along their axis (i.e., $r=5 \mathrm{~mm}$ and $\theta=0$ ). Then, applying Equation 1 to the difference of the two (opposite) voltages:

$$
\Phi_{1}-\Phi_{2} \approx \frac{2 p}{4 \pi \sigma(0.005)^{2}}
$$

Finally, rearranging to solve for the dipole moment $p$, we obtain:

$$
p \approx 2 \pi \sigma(0.005)^{2}\left(\Phi_{1}-\Phi_{2}\right)
$$

We use $\sigma=0.285 \mathrm{~S} / \mathrm{m}$, from the in vivo conductivity of human gray matter reported by Latikka et al. (2001).

\subsubsection{MEG source reconstruction}

We used NUTMEG (Dalal et al., 2004) to perform MEG source reconstruction. A multisphere head model (Huang et al., 1999) was computed for each patients' sensor configuration and head geometry. 
The timecourses of the neural generators of the evoked fields were reconstructed using a minimum variance beamformer (Van Veen et al., 1997). Beamformer weights were computed from the sample covariance of the unaveraged data and subsequently applied to the averaged sensor data to yield timecourses over a grid of voxels spanning the head volume. The results near electrode locations were compared with intracranial ERPs to assess data quality.

Separately, the oscillatory dynamics of the sources were reconstructed using a related time-frequency beamformer technique (Dalal et al., 2008a). This method outputs a spectrogram for each of the voxels spanning the head volume. The power bands chosen were $4-9 \mathrm{~Hz}, 9-25 \mathrm{~Hz}, 25-45 \mathrm{~Hz}, 55-95 \mathrm{~Hz}$, 105-145 Hz, and 155-195 Hz. The power line frequency of $50 \mathrm{~Hz}$ and its harmonics at $100 \mathrm{~Hz}$ and $150 \mathrm{~Hz}$ were avoided to reduce noise. As with the iEEG and sensor MEG spectrograms, post-stimulus power modulations were contrasted relative to the baseline period using Wilcoxon $Z$-scores.

\subsubsection{MEG sensitivity}

Finally, the multisphere head model was applied in order to estimate the sensitivity of MEG to locations probed with intracranial electrodes using the following procedure: A simulated unit dipole was placed at the midpoint between each bipolar electrode pair. As the orientation of sources detected with iEEG is generally uncertain - yet has a profound impact on MEG signal - we simulated a distribution of orientations, from $-\frac{\pi}{2}$ to $+\frac{\pi}{2}$ radians of elevation and ${ }^{-} \pi$ to ${ }^{+} \pi$ radians of azimuth, sampled every $\frac{\pi}{36}$ radians. The head model was used to calculate the theoretical MEG sensor distribution for each orientation at each brain location. The estimated magnetic fields for each sensor were then normalized by the maximum magnetic field (generated by a unit dipole anywhere in the brain) that could be detected by that sensor, yielding relative sensitivity (Malmivuo and Plonsey, 1995). Finally, for each dipole orientation at each brain location, the maximum relative sensitivity (i.e., the relative sensitivity at the most sensitive MEG sensor) was determined.

\section{Results}

\subsection{Evoked potentials and fields}

Figure 1 displays the event-related fields (MEG ERFs), beamformer source reconstructions, and potentials (iEEG ERPs) obtained by averaging directly the raw MEG and iEEG signals across trials for each condition. At all three levels of recording, evoked responses were clear and reproducible across condi- 
tions, with perhaps Pt2's source reconstruction suffering from increased noise. Intracerebral ERPs measured directly in the occipital and temporal lobes are shown together with the corresponding MEG scalp topographies, and the ERF trace at the sensor showing maximal response, for each patient and for both targets and distractors. In patients Pt1, Pt3, and Pt4, two consecutive bipolar pairs recorded ERPs with opposite polarities, indicating local sources. In all four patients, the MEG topography at the peak latency showed a clear amplitude peak over occipital sensors, consistent with the occipito-temporal responses found in iEEG.

\subsection{Stimulus-induced spectral energy modulations}

To compare oscillatory responses at the cortical and MEG level, we used a simple two-step strategy: a) use iEEG data to detect possible alpha, beta, and gamma band responses at the cortical level, and $b$ ) test for similar effects at the sensor level, at the same latencies and frequency range. At both levels, responses were defined as significant energy increases or decreases relative to the $\left[^{-} 200\right.$ to $\left.{ }^{-} 50 \mathrm{~ms}\right]$ pre-stimulus baseline (using Wilcoxon tests, as explained in the Methods section).

\subsection{1 iEEG recordings}

Clear gamma band responses (GBRs) to target words were observed in all four patients, distributed over the temporal, occipital, and parietal cortex, in both hemispheres (note that there were no electrodes in the frontal lobes) (Pt1: GBR in 14 out of 44 bipolar pairs; Pt2: 1/14; Pt3: 3/27; Pt4: 2/23) (see Figures $2-6$ and $\mathrm{S} 1$ for the precise locations of GBRs for target words on individual 3D MRI renderings). The time and frequency extents of those responses were remarkably consistent across sites and patients with their highest energy concentrated in the $[100-400 \mathrm{~ms}]$ time window. Some of those responses (Pt1: 6/14; Pt2: 0/1; Pt3: 2/3; Pt4: 0/2) were stronger for targets than for distractors (KW, $p<0.05$, corrected), while the reverse effect did not occur. Also, we found no cortical site producing GBRs solely for distractors; however, we observed some negative GBRs, i.e., gamma band suppressions (see Figure 7).

Frequencies in the alpha and beta bands (9-25 Hz) were characterized by a common pattern opposite to GBRs, with energy decreases between $200 \mathrm{~ms}$ and $500 \mathrm{~ms}$ (see Figures S2-S6). For statistical analysis, all frequencies in that range were pooled together, and significant energy reductions relative to the baseline were called alpha/beta suppressions (ABS). There was strong spatial correspondence between GBR and ABS: we found ABS in all cortical sites with $0-100 \mathrm{~Hz}$ GBR in addition to some adjacent sites (Pt1: 18/44; 
Pt2: 1/14; Pt3: 4/27; Pt4: 3/23, Wilcoxon $p<0.05$, corrected). As shown in Figures 7 and S1, along the same electrode probe, ABS typically extended 1-2 $\mathrm{cm}$ beyond the GBR. In Pt1, we observed that ABS was stronger for targets than for distractors in all locations showing ABS (Pt1: 18/44; KW $p<0.05$, corrected); there were no such effects in the other three patients.

The gamma band and the alpha/beta bands differed also in their synchronization properties: in patient Pt1, in which intracerebral GBR and ABS were found in several distant cortical sites, we compared the level of synchrony across those sites in the gamma and alpha/beta bands: A first test, which compared the mean synchrony value in a large $\left[{ }^{-} 200\right.$ to $\left.{ }^{+} 500 \mathrm{~ms}\right]$ time window for the 9-25 Hz vs. the 50-100 Hz range (cross-site synchrony being calculated and averaged across all 39 pairs of non-adjacent iEEG sites in this patient) revealed a higher synchrony in the 9-25 Hz range (Wilcoxon $Z=4.71, p<0.001)$. Yet, one may reasonably argue that synchrony should be measured for each frequency band when that band is really active (that is, post-stimulus for the gamma range, and pre-stimulus for the alpha/beta range, since ABS correspond to the disappearance of strong baseline oscillations). Therefore, we performed a second test, similar to the first one, but comparing the synchrony measured $a$ ) for the $9-25 \mathrm{~Hz}$ range, in the $\left[{ }^{-} 200\right.$ to $-50 \mathrm{~ms}$ ] baseline time-window vs. $b$ ) for the $50-100 \mathrm{~Hz}$ range in a window of same duration but chosen during maximal GBR: [200 to $350 \mathrm{~ms}]$. Again, synchrony was higher in the 9-25 $\mathrm{Hz}$ range (Wilcoxon $Z=3.21, p=0.001$ ). This result may partly explain the differences between MEG gamma band and alpha/beta band responses described below.

\subsubsection{MEG sensor spectrograms}

TF analysis of MEG data tested whether GBRs and ABS seen with iEEG were visible with MEG. Since iEEG responses were concentrated in the [100 to $400 \mathrm{~ms}$ ] time window (for GBR) and in the [200 to $500 \mathrm{~ms}$ ] time window (for ABS), the analysis evaluated differences in TF energy between the baseline and those two time windows, in both frequency ranges. Note that while iEEG GBRs extended over a broad range $(50-150 \mathrm{~Hz})$, visual inspection revealed that MEG GBRs were most evident in a narrower range $(50-100 \mathrm{~Hz})$, in agreement with previous MEG studies (Hoogenboom et al., 2006; Vidal et al., 2006). For this reason, 50-100 Hz was chosen for MEG GBR analyses, with the exception of patient Pt4, who only exhibited significant iEEG GBR in the 100-150 Hz range.

Figures 2-6 show topographical representations of the statistical energy variations relative to baseline (Wilcoxon $Z$-values) for MEG GBR. (See Figures S2$\mathrm{S} 6$ for ABS.) These figures also indicate sensors with significant energy variations using both uncorrected and Bonferroni-corrected thresholds $(p<0.05)$. 
Clear MEG GBRs were observed in the first patient, Pt1, over both hemispheres (50/151 sensors, uncorr., 26/151, corr.) and predominantly over the occipital sensors but extending over temporal and frontal regions (Figures 23). This topography was compatible with the cortical sources found around the temporo-parieto-occipital junction in this patient. In two other patients, Pt2 and Pt3, gamma band energy increases were much weaker, reaching significance level (uncorrected) in a relatively small number of sites (Pt2: 26/151, Pt3: 16/151). In patient Pt4, strong occipital MEG GBR was observed in the 50-100 Hz range (Figure S6) as well as 100-150 Hz (Figure 6), but intracranial electrodes covered frontal and anterior temporal regions.

Uncorrected statistical comparison between conditions revealed stronger GBR for targets in two patients (Pt1, 8/151 sensors, Pt2, 5/151 sensors); but one right frontal sensor in Pt1 had a stronger GBR for distractors. There was no difference between conditions in Pt3 and Pt4.

ABS was stronger than GBR at the MEG level. In all four patients, we found significant ABS, predominantly over the occipital sensors, in good agreement with the MEG suppression previously reported in the same frequency range (Hoogenboom et al., 2006) in response to visual stimuli. In contrast with GBR, most ABS were significant at both corrected and uncorrected thresholds. In three patients (Pt1, Pt2, and Pt4), ABS were also stronger for targets than for distractors (with both thresholds). This effect was not observed in Pt3, whose electrode placement did not allow a head position with full coverage of occipital regions.

Although strongest ABS and GBR were observed over the posterior sensors, we also found frontal responses (consistent with the involvement of frontal language areas, such as Broca's area, in reading). Focal GBR was observed over left frontal sensors in patients Pt1 and Pt4, spatially coinciding with ABS. In patient $\mathrm{Pt3}$, we also found an increase of alpha/beta activity (alpha/beta activation) over those regions in response to distractors, consistent with a deactivation of this region with less attention.

Since the strongest GBR were obtained in the patient with the largest number of analyzed trials (Pt1, 530 trials per condition compared to 210, 365, and 300 for the other three patients), we tested whether the number of trials may have had an effect on the GBR/ABS estimation. We checked whether strong GBRs were also apparent in this patient with less trials analyzed (Figure 8). Wilcoxon tests performed with 50, 100, 250, and 500 trials revealed that for both ABS and GBR, Wilcoxon $Z$ values increased with the number of trials, which was expected for mathematical reasons. However, the topography of $Z$ values varied considerably with the number of trials, especially for frontal GBR, even between 250 and 500 trials. Therefore, the number of trials in Pt2 (210 trials per condition) and Pt3 (365 per condition) may have been too small 
for stable GBR to emerge in the MEG topography given the SNR conditions in those patients. In contrast, this instability of the $Z$-score topography was not as pronounced for ABS.

\subsubsection{MEG source spectrograms}

Next, a time-frequency beamformer source reconstruction was applied to the MEG responses. All four subjects displayed strong, focal occipital GBR and ABS with a latency of $200-250 \mathrm{~ms}$, but only Pt1 had intracranial electrodes near this region.

Therefore, the brain location which showed the strongest GBR in iEEG were probed with the time-frequency beamformer for each patient. As with the iEEG and MEG sensors, the time-frequency maps reflect the statistical energy variations relative to baseline with Wilcoxon $Z$ values. GBR in the $55-95 \mathrm{~Hz}$ band was successfully recovered for the strongest GBR responses found in both the right and left hemispheres. This activity was not significantly modulated between the two conditions for the right hemisphere location (located in occipital cortex), in agreement with the iEEG results (Figure 2). In the left hemisphere, at a location in the posterior temporal lobe near the temporooccipital junction, displayed modulation of GBR with attention (Figure 3), also in agreement with iEEG results. (The GBR appeared to start earlier compared to the iEEG, but this activity was in fact spatial spread from the strong occipital GBR.) In both cases, significant activity was found in $55-95 \mathrm{~Hz}$, but not in higher frequency bands.

In patient Pt2, 55-95 Hz GBR was detected with a somewhat noisy time course (Figure 4), and in patient Pt3, not at all (Figure 5). Unexpectedly, a striking correspondence was found with iEEG in even higher frequency bands - 105-145 Hz for Pt2 and 145-195 Hz for Pt3. Significant GBR in the 105$145 \mathrm{~Hz}$ range was also found for the target condition in Pt4, in accordance with the iEEG results; however, the distractor responses appeared noisy and activity above $55 \mathrm{~Hz}$ was not found to be significant.

In all patients, significant $\mathrm{ABS}$ with frequency and duration in agreement with iEEG results were detected (Figures S2-S6). Additionally, significant GBR and ABS source reconstructions agreed with spatiotemporal patterns observed in a parallel 10-subject iEEG study from our group (see movie in Supplementary Material and Jung et al., 2008), providing corroborating evidence for brain locations that were not sampled by iEEG in the present study. 


\subsubsection{MEG sensitivity}

As a partial exploration of the mechanisms behind the strength of MEG GBR with respect to observed iEEG GBR, we estimated the MEG sensitivity for iEEG GBR locations (see Figure 9). Each column of the plot depicts the histogram distribution of MEG sensitivity over all possible source orientations for a given brain location known to exhibit GBR in iEEG. The half-sensitivity volume (HSV) is a common quantifier of MEG detectability, and includes the brain volume for which the relative sensitivity is greater than 0.5 for a given MEG sensor.

In Pt1, 5 of 14 GBR locations (identified with iEEG) fell within the halfsensitivity volume (HSV) for at least one MEG sensor, indicating favorable conditions for MEG detection. (Of the remaining electrodes, five were in deep locations in temporal cortex on probes ultimately reaching the hippocampi.) Interestingly, electrode TePg2-1 (near the left occipital pole) recorded the strongest dipole moment (see below) and was placed in the most MEGsensitive location; further exploration of the sensitivity analysis showed that left occipital and left posterior temporal sensors would be most sensitive to this source, in accordance with the observed results shown in Figure 3.

While iEEG detected only one significant GBR location in Pt2, this location was on the cortical surface, with a high median MEG sensitivity of 0.71. Even though very specific source orientations would considerably reduce the MEG sensitivity, the distribution shows that sensitivities were heavily concentrated above 0.6 over the range of all possible orientations.

Median MEG sensitivities for the 3 GBR locations found in Pt3 were all low, ranging from $0.33-0.43$, with only the "luckiest" possible orientations yielding sensitivities slightly over 0.5. As such, the difficulty in resolving GBR with Pt3 may be partly explained by this low sensitivity.

Finally, the orbitofrontal location in Pt4 showing 100-150 Hz activity also had a relatively low median sensitivity of 0.24 .

\subsubsection{GBR dipole moment estimation}

While the density of the iEEG electrodes was not sufficient to allow for a precise determination of current source density or dipole strength, we nevertheless attempted to obtain an estimate of the source magnitudes involved using Equation 3. The estimated GBR dipole moments ranged from 0.04$0.65 \mathrm{nAm}$ (see Figure 9, red diamonds), an order of magnitude below dipole moments estimated for averaged ERPs from the same data. Dipole strengths for gamma sites from Pt2, Pt3, and Pt4 were particularly low, compared to several sites from Pt1. While the iEEG dipole moments cannot be directly 
evaluated in the context of the magnetic field strength observed with MEG, we note that MEG sensor amplitudes were also an order of magnitude lower for GBR relative to averaged ERFs for all patients.

\section{Discussion}

Our primary objective was to test whether attention-related modulation of GBR and ABS observed with intracerebral measurements during a reading task could also be detected noninvasively with MEG. We found that this was indeed the case for the alpha/beta suppression, which was robust and consistent between the two attention conditions in all four patients, and was strengthened by attention in three patients in whom the occipital lobe was properly sampled by the MEG sensors (for practical reasons, one patient had to be positioned lower relative to the MEG helmet). By contrast, the GBR recorded in MEG and its modulation by attention, were not as strong and robust as those found in the simultaneously acquired intracerebral data.

\subsection{MEG gamma band responses}

GBR were clear at the sensor level in two patients, Pt1 and Pt4, reaching above the significance threshold even after Bonferroni correction. In another patient, Pt2, the responses were weaker and more sparse, always below the Bonferroni-corrected significance threshold; results were significantly stronger for targets in only two sensors, but more encouraging with the beamformer source reconstruction with more robust responses in the 105-145 $\mathrm{Hz}$ range. The evoked fields and corresponding source reconstructions were also much noisier for this patient than the others. In the remaining patient, Pt3, GBR seemed slightly stronger for distractors at the sensor level, although the statistical analysis revealed no significant difference between the two conditions; source reconstruction appeared to reveal modulations at unexpectedly high frequencies (155-195 Hz), but surprisingly not between 50-150 Hz. These results, together with the lack of resemblance between the gamma band topography in the two experimental conditions, indicate that we were dealing with effects barely above noise level in two out of four patients.

Two recent studies have called into question the origin of GBR in scalp EEG, with one attributing it to EMG of scalp muscles (Whitham et al., 2008) and the other to miniature saccades (Yuval-Greenberg et al., 2008). MEG is assumed to be subject to the same types of physiological noise. Since the present MEG data was simultaneously acquired with intracranial EEG, our results strongly suggest that no "false" GBR in regions with dense electrode coverage, i.e., 
MEG responses unexplained by iEEG. Furthermore, beamformer methods in particular do not assume cortical origin for all signals, and have indeed been shown to be robust in the presence of physiological noise sources external to the brain (Bardouille et al., 2006; Cheyne et al., 2006, 2007).

The technical difficulty of detecting GBR at the scalp level has been acknowledged and discussed thoroughly in recent studies (Trujillo et al., 2005; Hoogenboom et al., 2006). Still, a long list of EEG and more recently MEG studies on gamma band responses (i.e., Bauer et al., 2006; Düzel et al., 2003; Hall et al., 2005; Hoogenboom et al., 2006; Kaiser et al., 2004, 2002; Osipova et al., 2006; Sokolov et al., 1999; Vidal et al., 2006; Dalal et al., 2008a) attests that such difficulties can be overcome, given certain precautions that remain to be formalized. What can we learn from the present experiment? In their study, Hoogenboom et al. (2006) listed a series of reasons explaining why gamma band responses have been difficult to find in noninvasive recordings. Those reasons fall into two categories, related either to the experimental design (use of transient static stimuli non-optimal for generating GBR, paradigms which do not require the subject's sustained attention) or to the recording/analysis techniques (inadequate spectral analysis methods or recording apparatus). The authors proposed and clearly demonstrated, that with the proper paradigm and method, and with stimuli known to generate strong GBR at the cortical level in the primary visual cortex (e.g., high contrast, slow moving grids presented foveally under the subjects focused attention), MEG GBR were robust over the occipital sensors.

Conversely, one might propose that suboptimal stimuli (i.e., transient static stimuli) may fail to generate strong scalp EEG/MEG GBR simply because they do not generate strong GBR at the cortical level. Still, using stimuli quite different from Hoogenboom et al. (2006), and common to many ERP experiments with short and transient stimuli repeated at short intervals, we found strong GBR at the cortical level for two patients, but GBR was less clear in MEG. The weakness of those MEG GBR could therefore not be attributed to the first category of reasons mentioned by Hoogenboom et al. (2006) for weak scalp GBR. In fact, we also used the same MEG system as their group, with analysis techniques proven to be efficient to detect GBR in both scalp EEG (Tallon-Baudry et al., 1996) and MEG (Vidal et al., 2006; Dalal et al., 2008a), so the failure to detect clear MEG GBR in some of our data cannot be due to the reasons listed by Hoogenboom et al. (2006).

Several additional reasons can be proposed: $a$ ) choice of threshold for statistical significance, $b$ ) contamination of the gamma band by biological noise such as EMG, c) limited spatial extent of the GBR over the cortical surface and lack of synchrony between gamma band sources, $d$ ) source characteristics, such as strength, location, and orientation, $e$ ) existence of "negative" GBR, $f$ ) insufficient number of trials, $g$ ) frequency-dependent transmission of cortical 
signals, and $h$ ) sensor coverage and subject position in the MEG helmet.

Let us discuss those possible explanations:

a) statistical threshold: the Bonferroni correction used in this study is known to be too conservative, since statistical tests on neighbor sensors are far from being independent considering the limited spatial resolution of MEG. But, still, one source of dissatisfaction with MEG GBR was the lack of reproducibility, in terms of topography, between the two attention conditions in Pt2 and Pt3. Attention is known to modulate the activity within the networks processing sensory information, but not to change radically the organization of those networks; therefore, the general shape of the gamma band topography was expected to be similar between the two attention conditions, as for the intracranial evoked potentials.

b) biological noise: frequency-wise, gamma band responses overlap with several sources of noise, mostly from muscular origin, which do not affect the intracerebral signal. Since the degree of contamination of the MEG signals by muscle activity varies across patients, this might explain why MEG GBR were more visible in certain patients than others, according to their ability to relax this muscle activity. We therefore advocate the systematic use of simple biofeedback techniques (i.e., display of muscular activity) prior to the experiment to help subjects reduce this source of noise.

c) spatial extent and synchrony: since MEG signals arise from the summated activity of large cortical populations, they are, like scalp EEG, very sensitive to the degree of synchrony between those neural activities: synchronous oscillations summate into macro-oscillations at the sensor level, while uncorrelated or delayed-phase oscillations tend to cancel (Pfurtscheller and Cooper, 1975). Our synchrony analysis revealed that the degree of synchrony in the gamma range was indeed significantly smaller than in the alpha and beta range, where MEG oscillations were much more visible. Also, we showed that GBR tend to be less widespread than alpha and beta responses; this may constitute a problem for GBR produced by sources radial with respect to the skull surface, due to the reduced contribution to external magnetic fields.

d) GBR source characteristics: the magnitude of high-gamma band signal is generally 1-2 orders of magnitude smaller than the dominating content in lower frequencies. While this may still provide sufficient signal for intracranial recordings, the associated electric and magnetic fields decay with distance. Therefore, deeper, low-amplitude, and/or suboptimally oriented GBR sources, combined with interference from neighboring cortical patches, will reduce the likelihood of noninvasive detection. In our particular data, MEG was particularly sensitive to several of the locations where GBR was found in Pt1, and additionally the GBR sources appeared to have stronger dipole moments. In 
comparison, while the sensitivity to Pt2's GBR site was rather high, its apparent dipole moment was very low; in Pt3, both factors may have been at play, as the estimated dipole strength was relatively low in addition to being poorly situated for MEG detection.

e) gamma band suppression: our observation of decreased gamma band responses could constitute another reason for weak GBR in MEG, as localized decrease in gamma power would partially cancel, at the scalp level, gamma band enhancements. Gamma band suppression has been repeatedly observed in EEG, especially at the intracranial level (Lachaux et al., 2005).

f) number of trials: the strongest MEG GBR were observed with the dataset containing the largest number of viable trials (Pt1, 530 trials), while the results were not apparent when the analyses were instead conducted with less than 250 trials. In contrast, there were 210 and 365 analyzed trials in patients Pt2 and $\mathrm{Pt} 3$ respectively. Therefore, the number of trials was likely a strong factor in the different GBR results across our three subjects.

These results suggest that for static transient stimuli the detection of GBR may require more trials than sustained stimuli (in this case, over 500; the experimental design in Hoogenboom et al. (2006) used six blocks of 75 trials). Those numbers differ markedly from those found in classic ERP experimental designs, and may seem impracticably large when testing several experimental conditions. A common alternative to using such large numbers of trials is to perform group statistics across several subjects, as most EEG/MEG studies do (Kaiser et al., 2004; Vidal et al., 2006). Such group statistics can reveal patterns of GBR recurrent across several individuals, not necessarily apparent in all the individual subjects because of low SNR. However, this strategy was ill-suited for the present study, which aimed to provide quantitative measures of attention in individual subjects. Alternatively, new signal processing techniques may be able to resolve neural signals, including GBR, from a fewer number of trials (Zumer et al., 2007).

g) frequency-dependent propagation: It is commonly stated that the skull and scalp effectively act as a lowpass filter, thereby passing lower frequency signals with more gain than higher frequency signals. A handful of studies have attempted to quantify and explain the frequency dependence of cortex-to-scalp signal transmission. Some studies conclude that various head tissues have significant reactivity, i.e., the conductivity of various head tissues are frequencydependent even in the range of interest for EEG/MEG, with higher frequencies generally having higher conductivities than lower frequencies (Gabriel et al., 1996; Stinstra and Peters, 1998; Akhtari et al., 2003). The implications of this relationship for cortex-to-scalp signal transmission are not clear, but the simulations of Stinstra and Peters (1998) demonstrate that higher conductivities counterintuitively result in lower gains with a spherical head model, perhaps 
due to the effects of volume conduction. However, a study by Pfurtscheller and Cooper (1975) provides convincing evidence that the lowpass filter effect is primarily due to polyphasic summation, i.e., the phase variance of an activated cortical patch results in greater attenuation of higher-frequency signals when spatially averaged by a scalp EEG/MEG sensor. Unfortunately, such analysis of phase variance over cortical patches was not feasible with our electrode spacing and coverage.

h) sensor coverage and head position: An MEG system with denser sensor spacing may also increase the likelihood of recovering GBR, though this remains to be demonstrated; we are investigating the possibility of further simultaneous recordings of MEG and iEEG with a 275-channel system. Additionally, the weakest GBR were obtained for patient Pt3 whose brain was especially low relative to the helmet. For this patient, it is possible that coverage of the occipital and inferior temporal regions was incomplete, making the topography of both ABS and GBR difficult to interpret at the sensor level and reducing the SNR at the source level. This stressed the need to check the distance between the brain and the sensors before the experiment, a somewhat trivial, yet useful, precaution.

\section{2 $A B S$}

The topography of ABS was consistent across conditions, and the statistical differences between conditions were strong. Considering the difficulty sometimes associated with extracting MEG GBR, one can wonder why ABS were so much more clear than GBR in MEG data. The main reason is that the problems listed above for GBR do not apply to ABS. First, the signal to noise ratio in the alpha and beta bands is much better than in the gamma band, in part because the signal is stronger; it is well known that the spectrum energy of EEG and MEG data roughly decreases as frequency increases following a $1 / f$ law. Furthermore, noise sources are weaker, e.g., the spectrum of EMG artifact rarely extends below $25 \mathrm{~Hz}$ (Tallon et al., 1995). In addition, we showed that the synchrony level between distant sites was higher in the alpha/beta range than in the gamma range, in agreement with previous experimental (TallonBaudry et al., 2001; Lachaux et al., 2005) and theoretical studies (Kopell et al., 2000). Massively synchronous oscillations of this kind sum together in macroscopic MEG signals and are more visible at the sensor level; consequently, the post-stimulus suppression or desynchronization of these oscillations is also more visible in MEG signals. Finally, our data showed that ABS extended over wider cortical areas than GBR, a result consistent with previous intracranial studies (Crone et al., 1998a,b), and also found at the MEG level (Hoogenboom et al., 2006; Dalal et al., 2008a). This may also explain why the alpha/beta band modulations were more visible at the MEG level. ABS therefore consti- 
tutes a noninvasive index of attention during reading, which can be reliably measured even in single subjects. This further supports the role of ABS as a quantitative marker of attention (Palva and Palva, 2007; Hanslmayr et al., 2007).

A further interesting feature of ABS is that they often coincide with GBR at the cortical level. The precise nature of this relationship remains to be understood: most of the time, GBR seems to occur simultaneously with ABS (Crone et al., 1998a,b; Lachaux et al., 2005, 2006; Mainy et al., 2007), but the converse is not necessarily true, as shown here. Also, the reactivity of GBR and ABS to experimental manipulation seems to be somewhat different (Lachaux et al., 2005), but to an extent that is still undetermined. If the correlation is found to be systematic in certain cortical structures and experimental situations, ABS may constitute a convenient "tip of the iceberg" easily visible from scalp recordings and signaling the presence of underlying, less accessible GBR.

\subsection{Validation of MEG head models and source reconstruction algorithms}

The present data also provides a unique opportunity to test the relative performance of oscillatory source reconstruction algorithms. One important problem common to all methods is the accuracy of the head models used to estimate the lead field. We are currently investigating using these simultaneous recordings to empirically calculate the lead field and evaluate its impact on source reconstruction accuracy (Dalal et al., 2008b).

Finally, over the last few years, several algorithms have been proposed to reconstruct such sources and have been demonstrated to recover GBR in primary sensory cortices (Gross et al., 2001; David et al., 2003; Hall et al., 2005; Dalal et al., 2008a). Applications of these algorithms to more complex networks, while encouraging (van Wassenhove and Nagarajan, 2007; Guggisberg et al., 2008; Van Der Werf et al., 2008), are thus far scant. We have seen that the sources of gamma band responses in our task were multiple and widespread across the occipital, temporal, and parietal cortices; they should also involve language-related frontal areas, such as Broca's area and the precentral gyrus (Jung et al., 2008; Mainy et al., 2008). It would be particularly compelling to evaluate various MEG source reconstruction methodologies in the context of these complex activations. 


\section{Acknowledgments}

The authors would like to thank Florence Bouchet, Jean-Claude Bourzeix, and Laurent Hugueville for their technical help. JPL was supported by a grant from the Fondation Fyssen. SSD was supported by a Bourse Chateaubriand from the Government of France and a Marie Curie Fellowship from the European Commission.

\section{References}

Akhtari, M., Bryant, H. C., Emin, D., Merrifield, W., Mamelak, A. N., Flynn, E. R., Shih, J. J., Mandelkern, M., Matlachov, A., Ranken, D. M., Best, E. D., DiMauro, M. A., Lee, R. R., Sutherling, W. W., 2003. A model for frequency dependence of conductivities of the live human skull. Brain Topogr 16, 39-55.

Bardouille, T., Picton, T. W., Ross, B., 2006. Correlates of eye blinking as determined by synthetic aperture magnetometry. Clin Neurophysiol 117, 952-958.

Bauer, M., Oostenveld, R., Peeters, M., Fries, P., 2006. Tactile spatial attention enhances gamma-band activity in somatosensory cortex and reduces low-frequency activity in parieto-occipital areas. J Neurosci 26, 490-501.

Bichot, N. P., Rossi, A. F., Desimone, R., 2005. Parallel and serial neural mechanisms for visual search in macaque area V4. Science 308, 529-534.

Cheyne, D., Bakhtazad, L., Gaetz, W., 2006. Spatiotemporal mapping of cortical activity accompanying voluntary movements using an event-related beamforming approach. Hum Brain Mapp 27, 213-229.

Cheyne, D., Bostan, A. C., Gaetz, W., Pang, E. W., 2007. Event-related beamforming: a robust method for presurgical functional mapping using MEG. Clin Neurophysiol 118, 1691-1704.

Crone, N. E., Miglioretti, D. L., Gordon, B., Lesser, R. P., 1998a. Functional mapping of human sensorimotor cortex with electrocorticographic spectral analysis. II. Event-related synchronization in the gamma band. Brain 121, 2301-2315.

Crone, N. E., Miglioretti, D. L., Gordon, B., Sieracki, J. M., Wilson, M. T., Uematsu, S., Lesser, R. P., 1998b. Functional mapping of human sensorimotor cortex with electrocorticographic spectral analysis. I. Alpha and beta event-related desynchronization. Brain 121, 2271-2299.

Dalal, S. S., Guggisberg, A. G., Edwards, E., Sekihara, K., Findlay, A. M., Canolty, R. T., Berger, M. S., Knight, R. T., Barbaro, N. M., Kirsch, H. E., Nagarajan, S. S., 2008a. Five-dimensional neuroimaging: Localization of the time-frequency dynamics of cortical activity. NeuroImage 40, 1686-1700.

Dalal, S. S., Jerbi, K., Bertrand, O., Garnero, L., Baillet, S., Martinerie, J., Lachaux, J.-P., June 2008b. Experimental calculation of magnetic lead fields using MEG simultaneously acquired with intracranial EEG [abstract]. In: Proceedings of the 14th Annual Meeting of the Organization for Human Brain Mapping.

Dalal, S. S., Zumer, J. M., Agrawal, V., Hild, K. E., Sekihara, K., Nagarajan, S. S., 2004. NUTMEG: A neuromagnetic source reconstruction toolbox. Neurol Clin Neurophysiol, 52 . 
David, O., Cosmelli, D., Hasboun, D., Garnero, L., 2003. A multitrial analysis for revealing significant corticocortical networks in magnetoencephalography and electroencephalography. NeuroImage 20, 186-201.

Dormont, D., Cornu, P., Pidoux, B., Bonnet, A. M., Biondi, A., Oppenheim, C., Hasboun, D., Damier, P., Cuchet, E., Philippon, J., Agid, Y., Marsault, C., 1997. Chronic thalamic stimulation with three-dimensional MR stereotactic guidance. AJNR Am J Neuroradiol 18, 1093-1107.

Düzel, E., Habib, R., Schott, B., Schoenfeld, A., Lobaugh, N., McIntosh, A. R., Scholz, M., Heinze, H. J., 2003. A multivariate, spatiotemporal analysis of electromagnetic time-frequency data of recognition memory. NeuroImage 18, 185-197.

Engel, J., 1987. Outcome with respect to epileptic seizures. In: Engel, J. (Ed.), Surgical Treatment of the Epilepsies. Raven Press, New York, pp. 553-71.

Fries, P., Reynolds, J. H., Rorie, A. E., Desimone, R., 2001. Modulation of oscillatory neuronal synchronization by selective visual attention. Science 291, 1560-1563.

Gabriel, S., Lau, R. W., Gabriel, C., 1996. The dielectric properties of biological tissues: II. Measurements in the frequency range $10 \mathrm{~Hz}$ to $20 \mathrm{GHz}$. Phys Med Biol 41, 2251-2269.

Gross, J., Kujala, J., Hämäläinen, M., Timmermann, L., Schnitzler, A., Salmelin, R., 2001. Dynamic imaging of coherent sources: Studying neural interactions in the human brain. Proc Natl Acad Sci U S A 98, 694-699.

Gruber, T., Müller, M. M., Keil, A., Elbert, T., 1999. Selective visual-spatial attention alters induced gamma band responses in the human EEG. Clin Neurophysiol $110,2074-2085$.

Guggisberg, A. G., Dalal, S. S., Findlay, A. M., Nagarajan, S. S., 2008. Highfrequency oscillations in distributed neural networks reveal the dynamics of human decision making. Front Hum Neurosci 1, 14.

Hall, S. D., Holliday, I. E., Hillebrand, A., Singh, K. D., Furlong, P. L., Hadjipapas, A., Barnes, G. R., 2005. The missing link: Analogous human and primate cortical gamma oscillations. NeuroImage 26, 13-17.

Hanslmayr, S., Aslan, A., Staudigl, T., Klimesch, W., Herrmann, C. S., Bäuml, K.H., 2007. Prestimulus oscillations predict visual perception performance between and within subjects. NeuroImage 37, 1465-1473.

Hoogenboom, N., Schoffelen, J.-M., Oostenveld, R., Parkes, L. M., Fries, P., 2006. Localizing human visual gamma-band activity in frequency, time and space. NeuroImage 29, 764-773.

Huang, M. X., Mosher, J. C., Leahy, R. M., 1999. A sensor-weighted overlappingsphere head model and exhaustive head model comparison for MEG. Phys Med Biol 44, 423-440.

Jung, J., Mainy, N., Kahane, P., Minotti, L., Hoffmann, D., Bertrand, O., Lachaux, J.-P., 2008. The neural bases of attentive reading. Hum Brain Mapp 29, 11931206.

Kaiser, J., Bühler, M., Lutzenberger, W., 2004. Magnetoencephalographic gammaband responses to illusory triangles in humans. NeuroImage 23, 551-560.

Kaiser, J., Lutzenberger, W., Ackermann, H., Birbaumer, N., 2002. Dynamics of gamma-band activity induced by auditory pattern changes in humans. Cereb Cortex 12, 212-221.

Kim, D.-S., Ronen, I., Olman, C., Kim, S.-G., Ugurbil, K., Toth, L. J., 2004. Spatial 
relationship between neuronal activity and BOLD functional MRI. NeuroImage $21,876-885$.

Kirchberger, K., Hummel, C., Stefan, H., 1998. Postoperative multichannel magnetoencephalography in patients with recurrent seizures after epilepsy surgery. Acta Neurol Scand 98, 1-7.

Kopell, N., Ermentrout, G. B., Whittington, M. A., Traub, R. D., 2000. Gamma rhythms and beta rhythms have different synchronization properties. Proc Natl Acad Sci U S A 97, 1867-1872.

Lachaux, J.-P., Fonlupt, P., Kahane, P., Minotti, L., Hoffmann, D., Bertrand, O., Baciu, M., 2007. Relationship between task-related gamma oscillations and BOLD signal: New insights from combined fMRI and intracranial EEG. Hum Brain Mapp 28, 1368-1375.

Lachaux, J.-P., George, N., Tallon-Baudry, C., Martinerie, J., Hugueville, L., Minotti, L., Kahane, P., Renault, B., 2005. The many faces of the gamma band response to complex visual stimuli. NeuroImage 25, 491-501.

Lachaux, J.-P., Hoffmann, D., Minotti, L., Berthoz, A., Kahane, P., 2006. Intracerebral dynamics of saccade generation in the human frontal eye field and supplementary eye field. NeuroImage 30, 1302-1312.

Lachaux, J.-P., Lutz, A., Rudrauf, D., Cosmelli, D., Le Van Quyen, M., Martinerie, J., Varela, F., 2002. Estimating the time-course of coherence between single-trial brain signals: an introduction to wavelet coherence. Neurophysiol Clin 32, 157174.

Latikka, J., Kuurne, T., Eskola, H., 2001. Conductivity of living intracranial tissues. Phys Med Biol 46, 1611-1616.

Mainy, N., Jung, J., Baciu, M., Kahane, P., Schoendorff, B., Minotti, L., Hoffmann, D., Bertrand, O., Lachaux, J.-P., 2008. Cortical dynamics of word recognition. Hum Brain Mapp 29, 1215-1230.

Mainy, N., Kahane, P., Minotti, L., Hoffmann, D., Bertrand, O., Lachaux, J.-P., 2007. Neural correlates of consolidation in working memory. Hum Brain Mapp 28, 183-193.

Malmivuo, J., Plonsey, R., 1995. Bioelectromagnetism-Principles and Applications of Bioelectric and Biomagnetic Fields. Oxford University Press, New York.

Mikuni, N., Nagamine, T., Ikeda, A., Terada, K., Taki, W., Kimura, J., Kikuchi, H., Shibasaki, H., 1997. Simultaneous recording of epileptiform discharges by MEG and subdural electrodes in temporal lobe epilepsy. NeuroImage 5, 298-306.

Müller, M. M., Gruber, T., Keil, A., 2000. Modulation of induced gamma band activity in the human EEG by attention and visual information processing. Int $\mathrm{J}$ Psychophysiol 38, 283-299.

Niessing, J., Ebisch, B., Schmidt, K. E., Niessing, M., Singer, W., Galuske, R. A. W., 2005. Hemodynamic signals correlate tightly with synchronized gamma oscillations. Science 309, 948-951.

Nobre, A. C., Allison, T., McCarthy, G., 1998. Modulation of human extrastriate visual processing by selective attention to colours and words. Brain 121 ( $\mathrm{Pt} 7$ ), $1357-1368$.

Oishi, M., Otsubo, H., Kameyama, S., Morota, N., Masuda, H., Kitayama, M., Tanaka, R., 2002. Epileptic spikes: Magnetoencephalography versus simultaneous electrocorticography. Epilepsia 43, 1390-1395. 
Osipova, D., Takashima, A., Oostenveld, R., Fernández, G., Maris, E., Jensen, O., 2006. Theta and gamma oscillations predict encoding and retrieval of declarative memory. J Neurosci 26, 7523-7531.

Palva, S., Palva, J. M., 2007. New vistas for alpha-frequency band oscillations. Trends Neurosci 30, 150-158.

Pfurtscheller, G., Cooper, R., 1975. Frequency dependence of the transmission of the EEG from cortex to scalp. Electroencephalogr Clin Neurophysiol 38, 93-96.

Rodriguez, E., George, N., Lachaux, J. P., Martinerie, J., Renault, B., Varela, F. J., 1999. Perception's shadow: Long-distance synchronization of human brain activity. Nature 397, 430-433.

Sederberg, P. B., Kahana, M. J., Howard, M. W., Donner, E. J., Madsen, J. R., 2003. Theta and gamma oscillations during encoding predict subsequent recall. J Neurosci 23, 10809-10814.

Shigeto, H., Morioka, T., Hisada, K., Nishio, S., Ishibashi, H., Kira, D.-i., Tobimatsu, S., Kato, M., 2002. Feasibility and limitations of magnetoencephalographic detection of epileptic discharges: Simultaneous recording of magnetic fields and electrocorticography. Neurol Res 24, 531-536.

Singer, W., 1999. Neuronal synchrony: a versatile code for the definition of relations? Neuron 24, 49-65, 111.

Sokolov, A., Lutzenberger, W., Pavlova, M., Preissl, H., Braun, C., Birbaumer, N., 1999. Gamma-band MEG activity to coherent motion depends on task-driven attention. NeuroReport 10, 1997-2000.

Steinmetz, P. N., Roy, A., Fitzgerald, P. J., Hsiao, S. S., Johnson, K. O., Niebur, E., 2000. Attention modulates synchronized neuronal firing in primate somatosensory cortex. Nature 404, 187-190.

Stinstra, J. G., Peters, M. J., 1998. The volume conductor may act as a temporal filter on the ECG and EEG. Med Biol Eng Comput 36, 711-716.

Sutherling, W. W., Akhtari, M., Mamelak, A. N., Mosher, J., Arthur, D., Sands, S., Weiss, P., Lopez, N., DiMauro, M., Flynn, E., Leah, R., 2001. Dipole localization of human induced focal afterdischarge seizure in simultaneous magnetoencephalography and electrocorticography. Brain Topogr 14, 101-116.

Szurhaj, W., Bourriez, J.-L., Kahane, P., Chauvel, P., Mauguière, F., Derambure, P., 2005. Intracerebral study of gamma rhythm reactivity in the sensorimotor cortex. Eur J Neurosci 21, 1223-1235.

Tallon, C., Bertrand, O., Bouchet, P., Pernier, J., 1995. Gamma-range activity evoked by coherent visual stimuli in humans. Eur J Neurosci 7, 1285-1291.

Tallon-Baudry, C., Bertrand, O., 1999. Oscillatory gamma activity in humans and its role in object representation. Trends Cogn Sci 3, 151-162.

Tallon-Baudry, C., Bertrand, O., Delpuech, C., Pernier, J., 1996. Stimulus specificity of phase-locked and non-phase-locked $40 \mathrm{~Hz}$ visual responses in human. J Neurosci 16, 4240-4249.

Tallon-Baudry, C., Bertrand, O., Fischer, C., 2001. Oscillatory synchrony between human extrastriate areas during visual short-term memory maintenance. J Neurosci 21:RC177, 1-5.

Tallon-Baudry, C., Bertrand, O., Hénaff, M.-A., Isnard, J., Fischer, C., 2005. Attention modulates gamma-band oscillations differently in the human lateral occipital cortex and fusiform gyrus. Cereb Cortex 15, 654-662. 
Tanji, K., Suzuki, K., Delorme, A., Shamoto, H., Nakasato, N., 2005. High-frequency gamma-band activity in the basal temporal cortex during picture-naming and lexical-decision tasks. J Neurosci 25, 3287-3293.

Trujillo, L. T., Peterson, M. A., Kaszniak, A. W., Allen, J. J. B., 2005. EEG phase synchrony differences across visual perception conditions may depend on recording and analysis methods. Clin Neurophysiol 116, 172-189.

Van Der Werf, J., Jensen, O., Fries, P., Medendorp, W. P., 2008. Gamma-band activity in human posterior parietal cortex encodes the motor goal during delayed prosaccades and antisaccades. J Neurosci 28, 8397-8405.

Van Veen, B. D., van Drongelen, W., Yuchtman, M., Suzuki, A., 1997. Localization of brain electrical activity via linearly constrained minimum variance spatial filtering. IEEE Trans Biomed Eng 44, 867-880.

van Wassenhove, V., Nagarajan, S. S., 2007. Auditory cortical plasticity in learning to discriminate modulation rate. J Neurosci 27, 2663-2672.

Vidal, J. R., Chaumon, M., O'Regan, J. K., Tallon-Baudry, C., 2006. Visual grouping and the focusing of attention induce gamma-band oscillations at different frequencies in human magnetoencephalogram signals. J Cogn Neurosci 18, 18501862.

Whitham, E. M., Lewis, T., Pope, K. J., Fitzgibbon, S. P., Clark, C. R., Loveless, S., Delosangeles, D., Wallace, A. K., Broberg, M., Willoughby, J. O., 2008. Thinking activates EMG in scalp electrical recordings. Clin Neurophysiol 119, 1166-1175.

Yuval-Greenberg, S., Tomer, O., Keren, A. S., Nelken, I., Deouell, L. Y., 2008. Transient induced gamma-band response in EEG as a manifestation of miniature saccades. Neuron 58, 429-441.

Zumer, J. M., Attias, H. T., Sekihara, K., Nagarajan, S. S., 2007. A probabilistic algorithm integrating source localization and noise suppression for MEG and EEG data. NeuroImage 37, 102-115. 


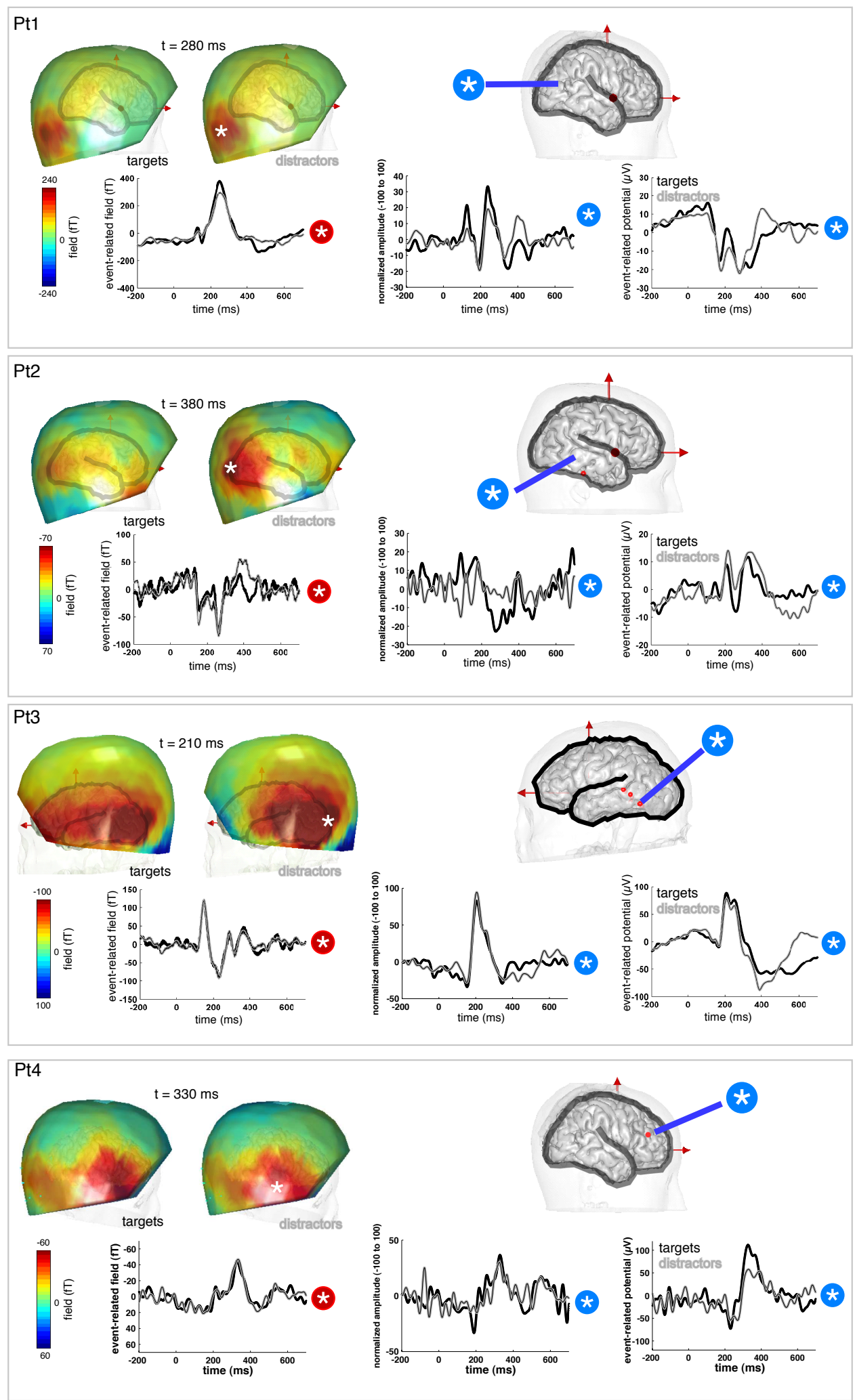

Fig. 1. MEG ERFs, MEG ERF beamformer reconstructions, and intracerebral ERPs. The sensor maps in the left column show the topography of the MEG evoked fields at the peak latency, for targets and distractors, together with the time series of the evoked fields measured at the peak ${ }_{7}$ sensor. The righthand column shows the evoked potential recorded at the intracerebral level in response to targets and distractors at sites highlighted on the 3D brain rendering. Finally, the middle column shows the corresponding MEG beamformer source reconstruction for those same sites. 


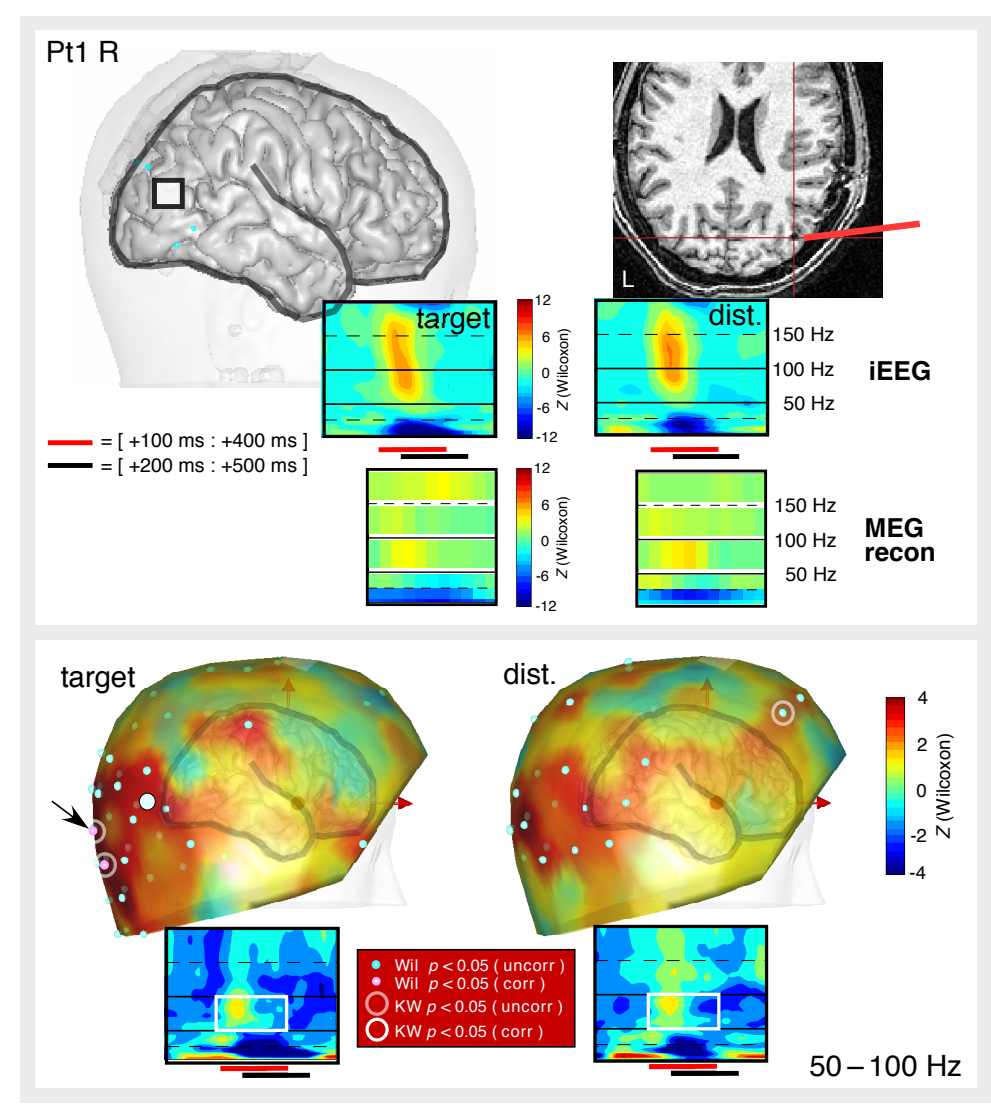

Fig. 2. MEG and iEEG GBR and ABS in patient Pt1 (right hemisphere). Top panel: MRI slice and 3D reconstruction showing a cortical site where significant GBR and ABS were observed in iEEG. The time-frequency maps show the result of the Wilcoxon comparison with the pre-stimulus baseline ( $\mathrm{TF} Z$ value map), for targets (left maps) and distractors (right maps), with the upper two maps computed from iEEG, while the lower two maps are computed from the MEG beamformer reconstructions for the same location. (Note beamformer timecourses start at $50 \mathrm{~ms}$, since no result can be computed for baseline windows.) Bottom panel: topographic Wilcoxon $Z$ maps at the MEG sensor level, for targets (left) and distractors (right) for the $[100 \mathrm{~ms}-400 \mathrm{~ms}] \times[50-100 \mathrm{~Hz}] \mathrm{TF}$ window (white box) compared with the baseline. The colormap was scaled such that it saturates in red for $Z$ values corresponding to $p=0.05$ (corrected). The adjacent TF maps show the Wilcoxon TF $Z$ maps for both conditions for the designated site (arrow). Blue dots indicate sensors with significant GBR using an uncorrected threshold, with pink dots using a corrected threshold. In circled sensors on the left (resp. right) topography, GBR was stronger for targets (resp. distractors) according to Kruskal-Wallis tests. White (resp. light gray) circles are for corrected (resp. uncorrected) thresholds. See Supplementary Material for corresponding ABS figures. 


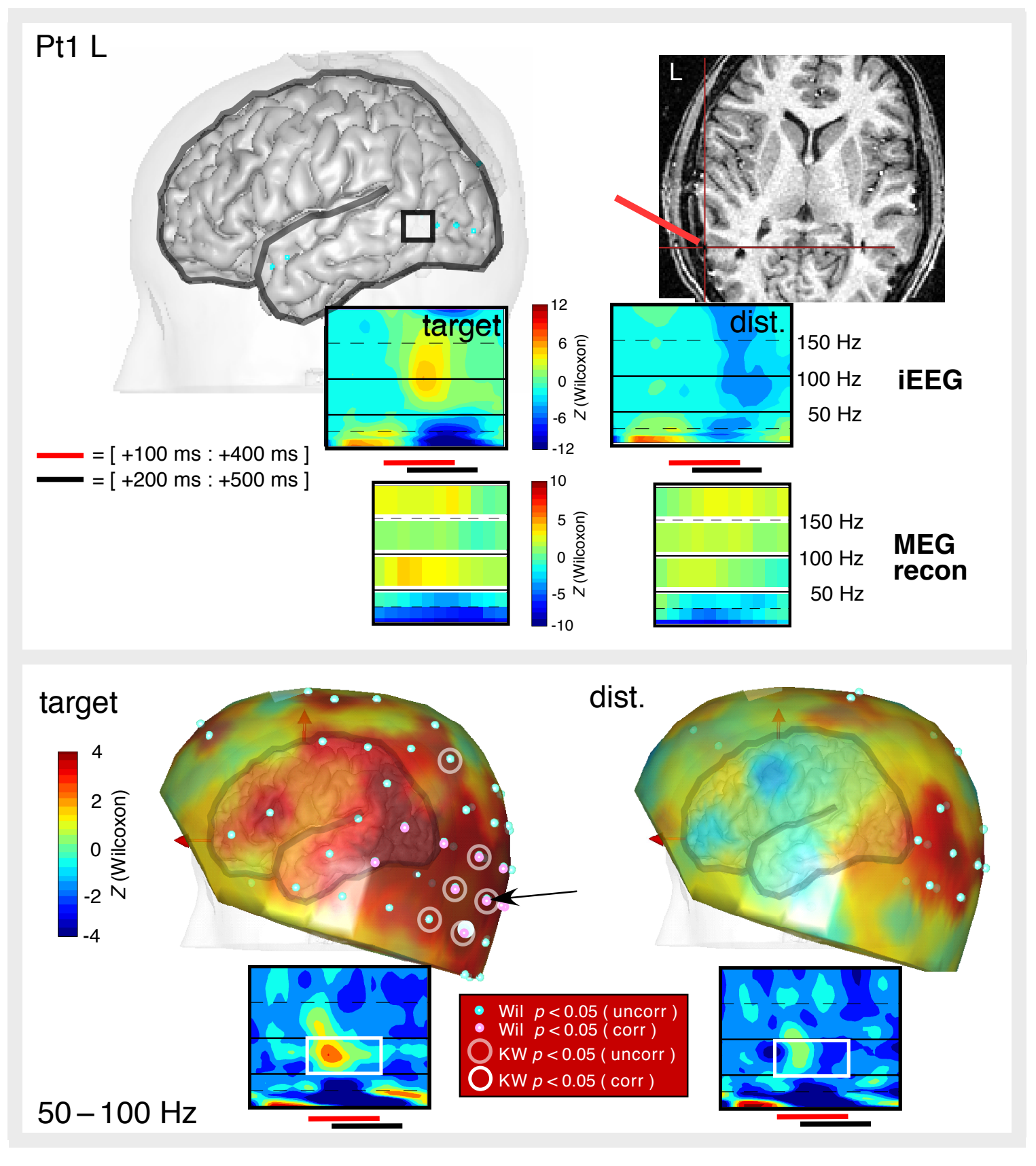

Fig. 3. Similar to Figure 2, for the left hemisphere of patient Pt1 


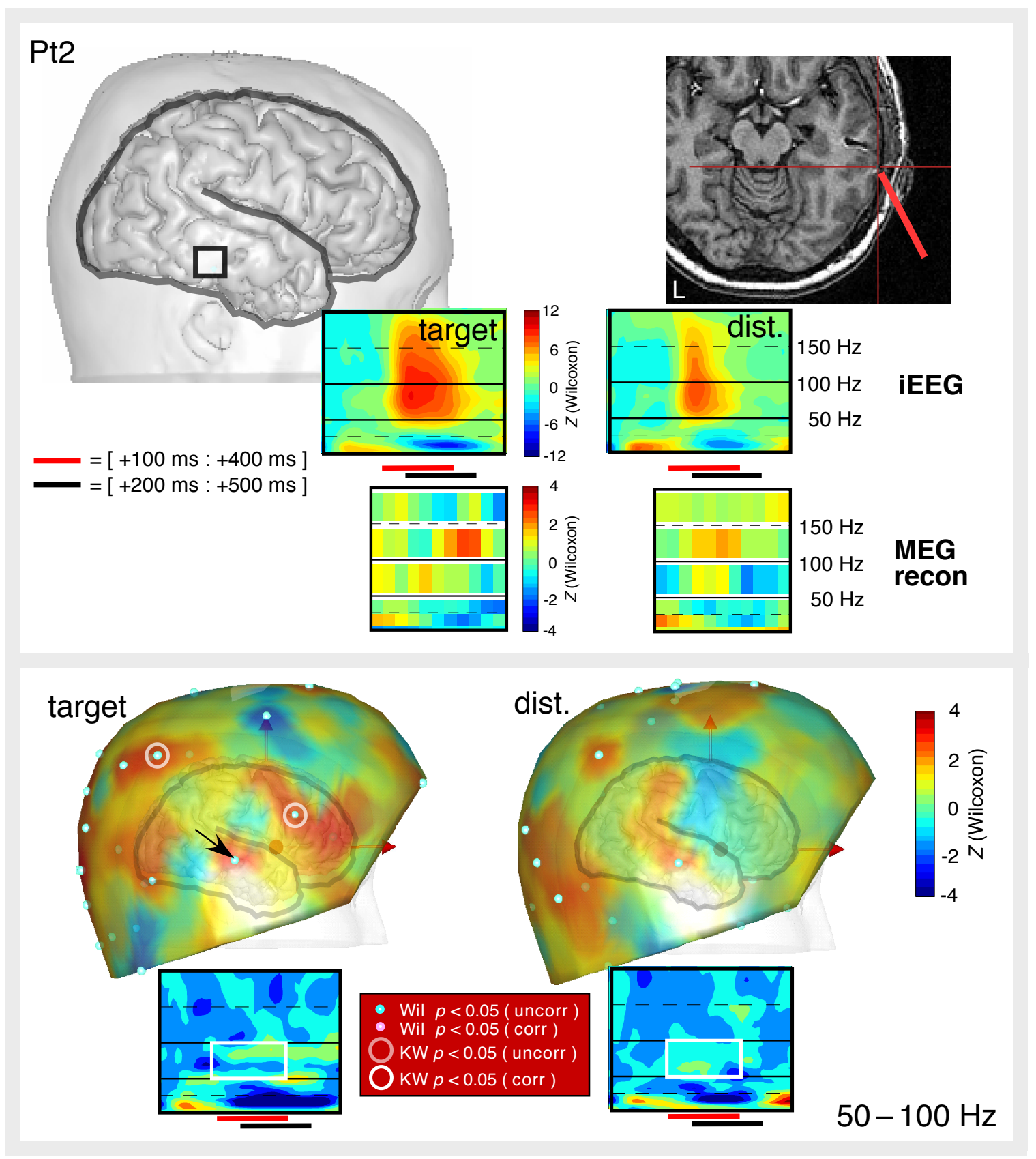

Fig. 4. Similar to Figure 2, for patient Pt2 


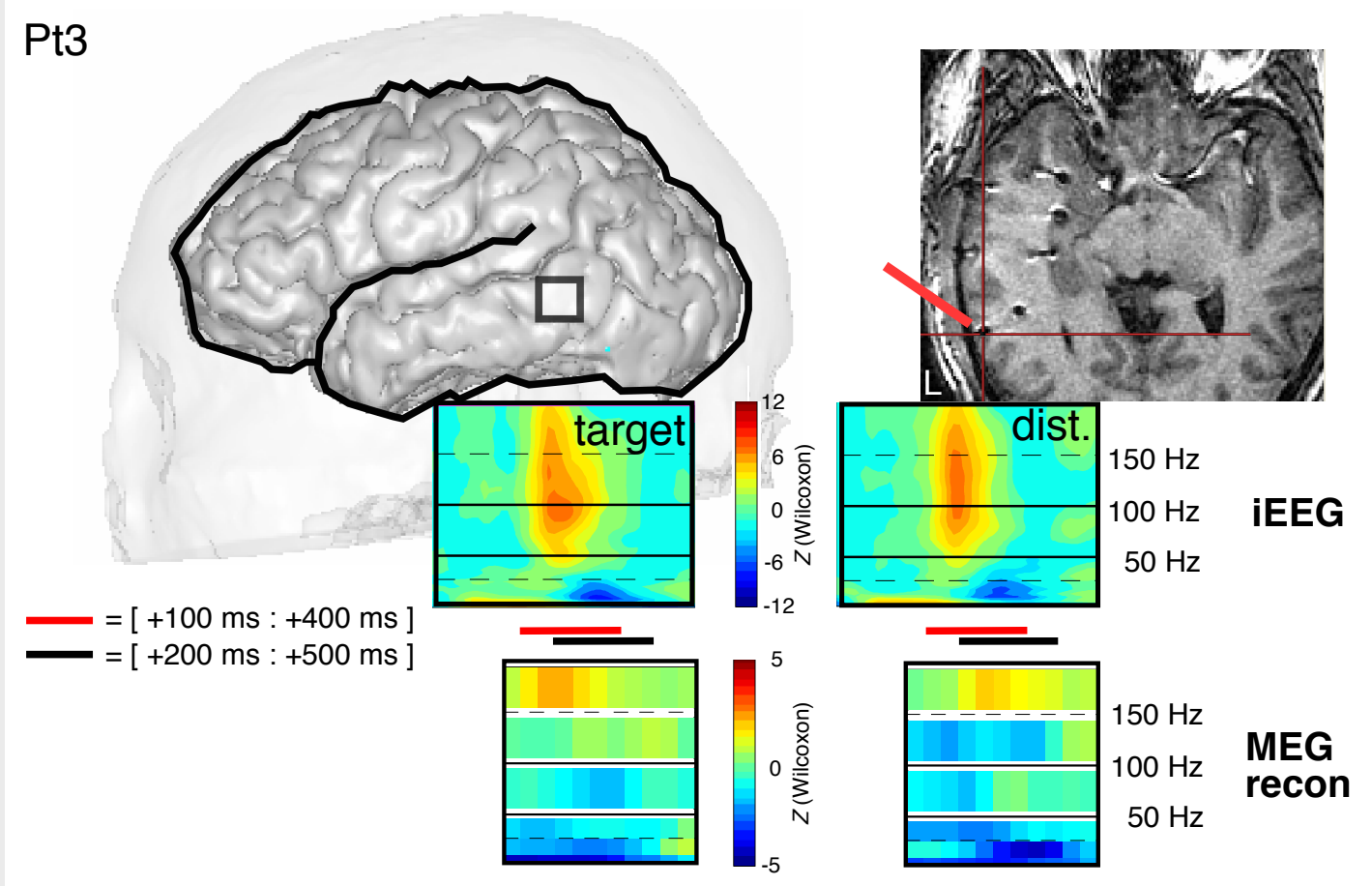

target
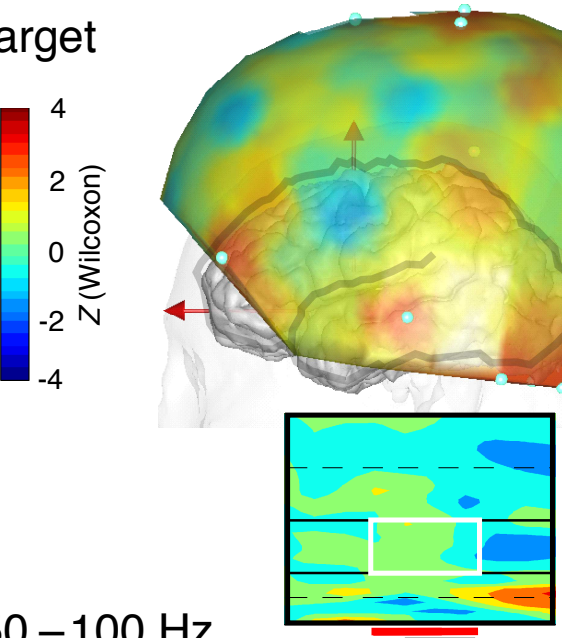

$50-100 \mathrm{~Hz}$ dist.
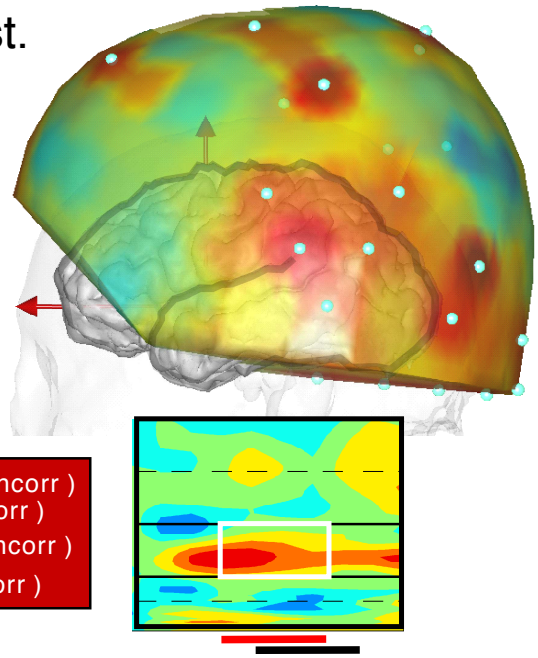

Fig. 5. Similar to Figure 2, for patient Pt3 


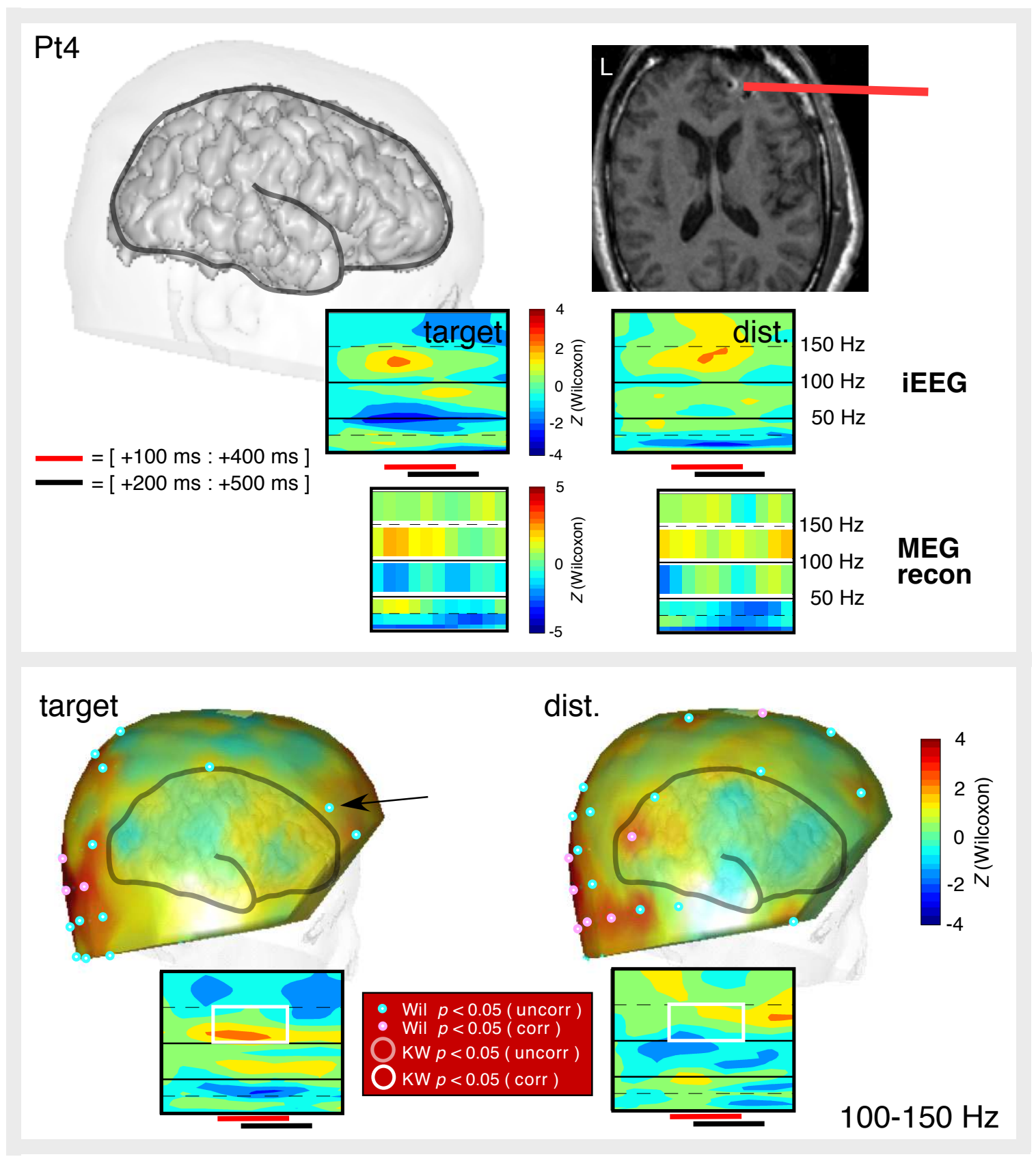

Fig. 6. Similar to Figure 2, for patient Pt4 


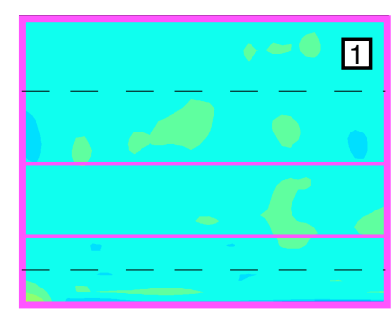

2
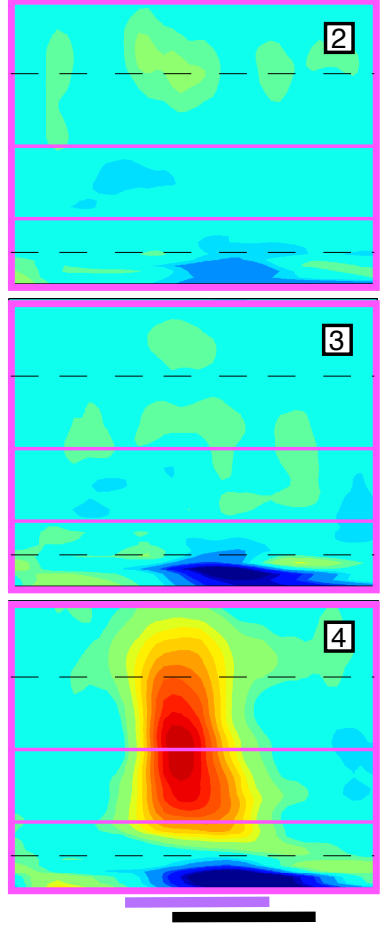

4

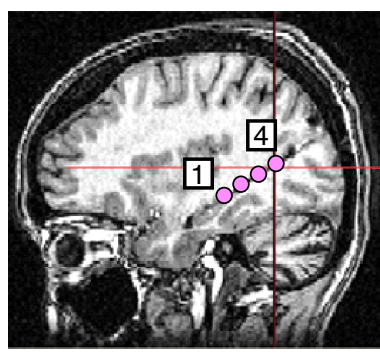

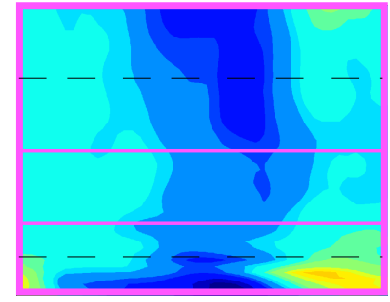
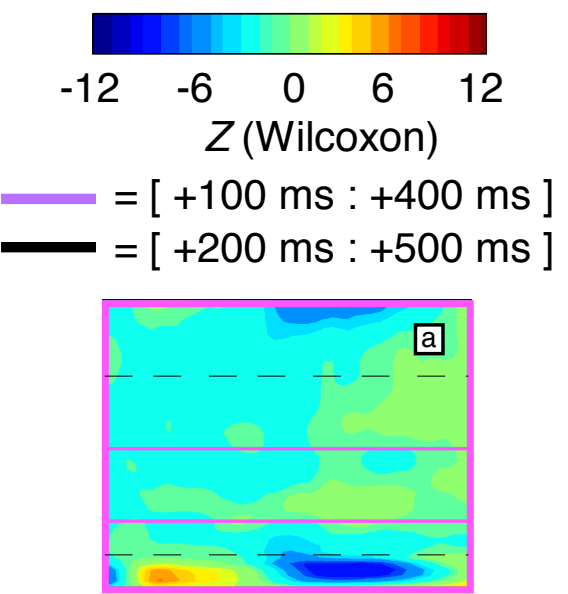

b
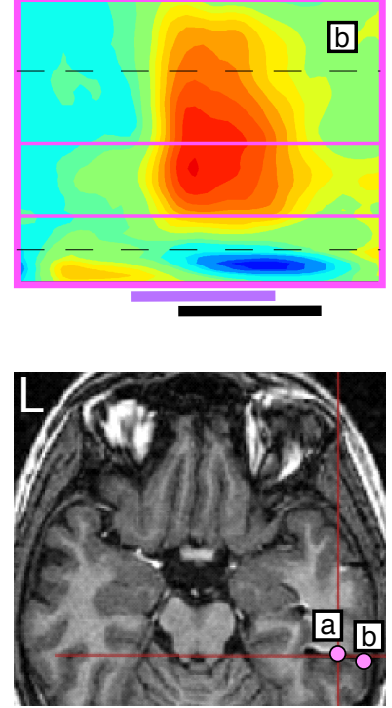

Fig. 7. Spatial extent of GBR and ABS. The TF maps show Wilcoxon $Z$ values for target words along two depth electrode arrays in two different patients. Moving from one site to the next along those electrodes, the ABS (in blue) appears more gradually than the GBR (in red). The TF Wilcoxon $Z$ map on the top right illustrates an example of negative GBR or gamma band suppression (statistical comparison with the $\left[{ }^{-} 200-{ }^{-} 50 \mathrm{~ms}\right]$ pre-stimulus baseline). 

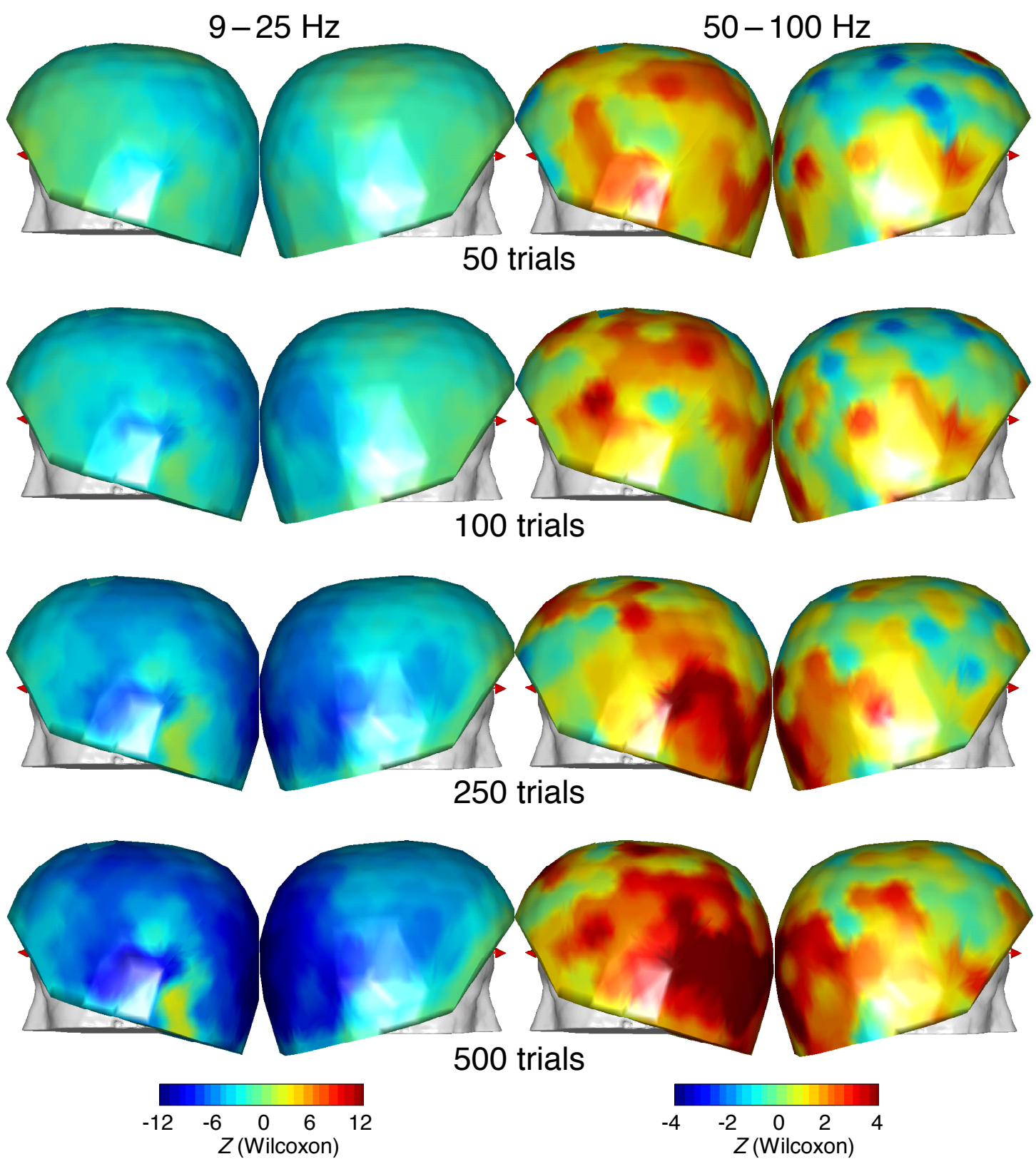

Fig. 8. Effect of number of trials on the detection of GBR and ABS. The topographical Wilcoxon $Z$ maps show for Pt1 the result of the comparison of the alpha/beta (left) and gamma (right) energy with baseline level (energy being measured in the $[200-500 \mathrm{~ms}] \times[9-25 \mathrm{~Hz}] \mathrm{TF}$ window for ABS and in the $[100-400 \mathrm{~ms}] \times[50-$ $100 \mathrm{~Hz}] \mathrm{TF}$ window for GBR). Maps were computed from 50, 100, 250, and 500 target stimuli; note how the topographical stability differs between the two frequency bands 


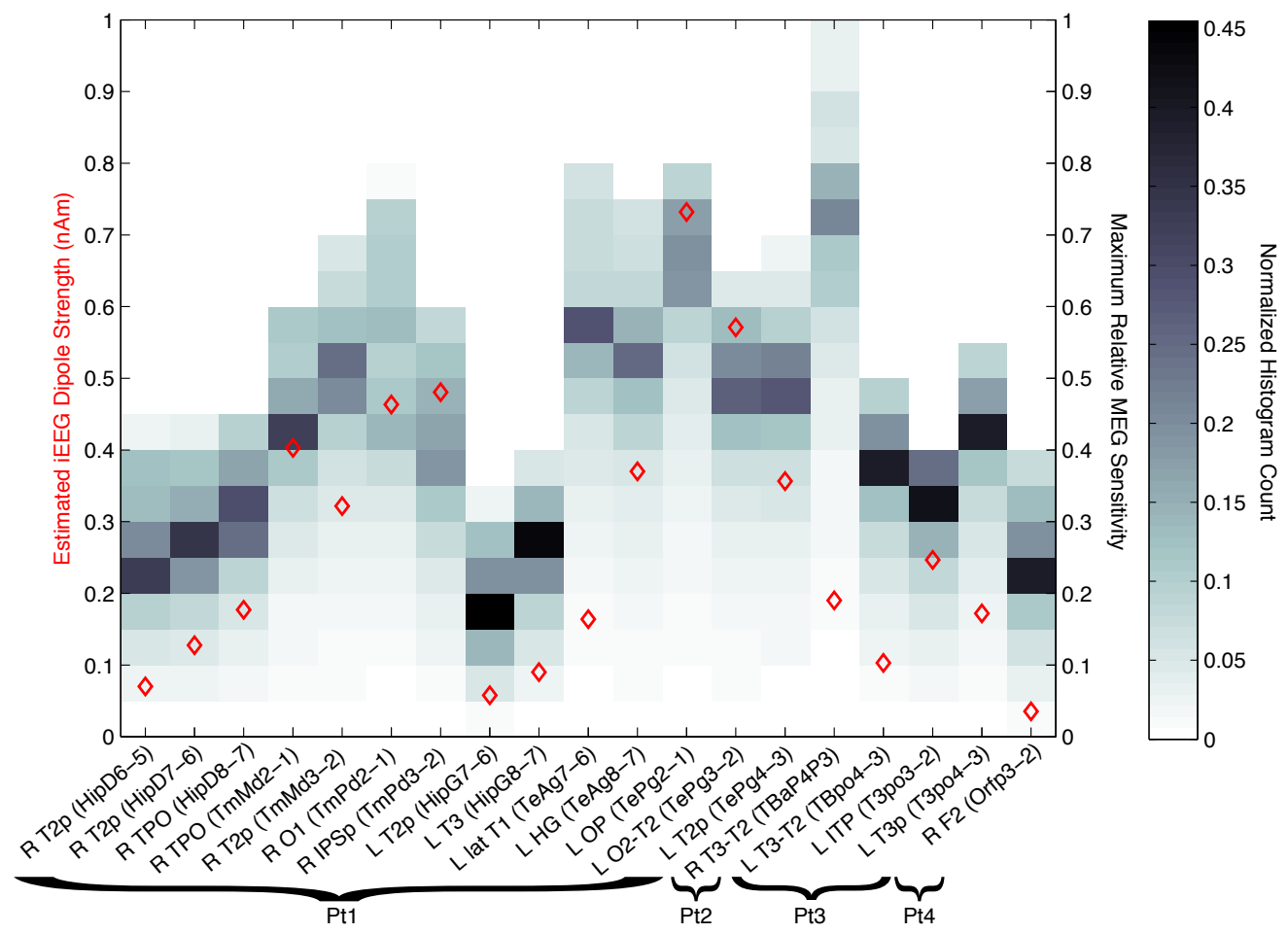

Fig. 9. An assessment of the MEG sensitivity to each GBR site revealed by iEEG. The relative sensitivity at a given MEG sensor is defined as the theoretical signal intensity for a unit dipole source at the electrode location normalized by the maximum intensity possible for any brain source; the maximum relative sensitivity is then the highest ratio found over the entire MEG sensor array for each GBR location. As the orientations of the GBR sources are unknown, we plot the distribution of maximum relative sensitivities for all 3D orientations (sampled at increments of $\pi / 36$ radians along azimuth and elevation). Therefore, the monochrome shading in each column represents a histogram of maximum sensitivities for each GBR location. The red diamonds indicate the GBR dipole moment estimated from the iEEG recordings; sites that have both a high sensitivity and a strong dipole moment are more likely to be detected with MEG. $\mathrm{L}=$ left; $\mathrm{R}=$ right; $\mathrm{T} 1=$ superior temporal gyrus; $\mathrm{T} 2$ $=$ middle temporal gyrus; $\mathrm{T} 2 \mathrm{p}=$ posterior middle temporal gyrus; $\mathrm{T} 3 \mathrm{p}=$ posterior inferior temporal gyrus; F2 = orbitofrontal cortex; ITP = inferior temporal pole; $\mathrm{TPO}=$ temporo-parieto-occipital junction; $\mathrm{O} 1=$ superior occipital gyrus; $\mathrm{OP}=$ occipital pole; IPSp = posterior intraparietal sulcus; HG = Heschl's gyrus 